\title{
STAR FORMATION LAWS IN BOTH GALACTIC MASSIVE CLUMPS AND EXTERNAL GALAXIES : EXTENSIVE STUDY WITH DUST CONINUUM, HCN (4-3), AND CS (7-6)
}

\author{
Liu, Tie
}

2016-10-01

Liu , T , Kim , K-T , Yoo , H , Liu , S-Y, Tatematsu , K, Qin , S-L , Zhang , Q, Wu , Y, Wang , K, Goldsmith , P F , Juvela, M , Lee , J-E , Toth , L V , Mardones , D , Garay , G , Bronfman , L, Cunningham , M R , Li , D , Lo , N , Ristorcelli , I \& Schnee , S 2016 , ' STAR FORMATION LAWS IN BOTH GALACTIC MASSIVE CLUMPS AND EXTERNAL GALAXIES : EXTENSIVE STUDY WITH DUST CONINUUM, HCN (4-3), AND CS (7-6) ' , Astrophysical Journal , vol. 829 , no. 2 , 59 . https://doi.org/10.3847/0004-637X/829/2/59

http://hdl.handle.net/10138/183621

https://doi.org/10.3847/0004-637X/829/2/59

unspecified

publishedVersion

Downloaded from Helda, University of Helsinki institutional repository.

This is an electronic reprint of the original article.

This reprint may differ from the original in pagination and typographic detail.

Please cite the original version. 


\title{
STAR FORMATION LAWS IN BOTH GALACTIC MASSIVE CLUMPS AND EXTERNAL GALAXIES: EXTENSIVE STUDY WITH DUST CONINUUM, HCN (4-3), AND CS (7-6)
}

\author{
Tie Liu ${ }^{1}$, Kee-Tae Kim ${ }^{1}$, Hyunju Yoo ${ }^{1,2}$, Sheng-Yuan Liu ${ }^{3}$, Ken’ichi Tatematsu ${ }^{4}$, Sheng-Li Qin ${ }^{5}$, Qizhou Zhang ${ }^{6}$, \\ Yuefang $\mathrm{Wu}^{7}, \mathrm{Ke} \mathrm{Wang}^{8}$, Paul F. Goldsmith ${ }^{9}$, Mika Juvela ${ }^{10}$, Jeong-Eun Lee ${ }^{11}$, L. Viktor Tóth ${ }^{12}$, Diego Mardones ${ }^{13}$, \\ Guido Garay ${ }^{13}$, Leonardo Bronfman ${ }^{13}$, Maria R. Cunningham ${ }^{14}$, Di $\mathrm{LI}^{15,16}$, Nadia Lo ${ }^{13}$, Isabelle Ristorcelli ${ }^{17}$, AND \\ SCOTT SCHNEE ${ }^{18}$ \\ ${ }^{1}$ Korea Astronomy and Space Science Institute 776, Daedeokdae-ro, Yuseong-gu, Daejeon 34055, Korea; liutiepku@ gmail.com \\ ${ }^{2}$ Department of Astronomy and Space Science, Chungnam National University, Daejeon, Korea \\ ${ }^{3}$ Academia Sinica, Institute of Astronomy and Astrophysics, P.O. Box 23-141, Taipei 106, Taiwan \\ ${ }^{4}$ National Astronomical Observatory of Japan, National Institutes of Natural Sciences, 2-21-1 Osawa, Mitaka, Tokyo 181-8588, Japan \\ ${ }^{5}$ Department of Astronomy, Yunnan University, and Key Laboratory of Astroparticle Physics of Yunnan Province, Kunming, 650091, China \\ ${ }^{6}$ Harvard-Smithsonian Center for Astrophysics, 60 Garden Street, Cambridge, MA 02138, USA \\ ${ }^{7}$ Department of Astronomy, Peking University, Beijing 100871, China \\ ${ }^{8}$ European Southern Observatory, Karl-Schwarzschild-Str.2, D-85748 Garching bei München, Germany \\ 9 Jet Propulsion Laboratory, California Institute of Technology, 4800 Oak Grove Drive, Pasadena, CA 91109, USA \\ ${ }^{10}$ Department of physics, University of Helsinki, FI-00014 Helsinki, Finland \\ ${ }^{11}$ School of Space Research, Kyung Hee University, Yongin-Si, Gyeonggi-Do 446-701, Korea \\ ${ }^{12}$ Department of Astronomy of the Loránd Eötvös University, Pázmány Péter sétány 1, 1117 Budapest, Hungary \\ ${ }_{14}^{13}$ Departamento de Astronomía, Universidad de Chile, Casilla 36-D, Santiago, Chile \\ ${ }^{14}$ School of Physics, University of New South Wales, Sydney, NSW 2052, Australia \\ ${ }_{16}^{15}$ National Astronomical Observatories, Chinese Academy of Sciences, Beijing, 100012, China \\ ${ }^{16}$ Key Laboratory of Radio Astronomy, Chinese Academy of Science, Nanjing 210008, China \\ ${ }^{17}$ IRAP, CNRS (UMR5277), Université Paul Sabatier, 9 avenue du Colonel Roche, BP 44346, F-31028, Toulouse Cedex 4, France \\ ${ }^{18}$ National Radio Astronomy Observatory, 520 Edgemont Road, Charlottesville, VA 22903, USA \\ Received 2016 January 28; revised 2016 August 14; accepted 2016 August 15; published 2016 September 22
}

\begin{abstract}
We observed 146 Galactic clumps in HCN (4-3) and CS (7-6) with the Atacama Submillimeter Telescope Experiment $10 \mathrm{~m}$ telescope. A tight linear relationship between star formation rate and gas mass traced by dust continuum emission was found for both Galactic clumps and the high redshift $(z>1)$ star forming galaxies (SFGs), indicating a constant gas depletion time of $\sim 100 \mathrm{Myr}$ for molecular gas in both Galactic clumps and high $z$ SFGs. However, low $z$ galaxies do not follow this relation and seem to have a longer global gas depletion time. The correlations between total infrared luminosities $\left(L_{\mathrm{TIR}}\right)$ and molecular line luminosities $\left(L_{\mathrm{mol}}^{\prime}\right)$ of HCN (4-3) and CS (7-6) are tight and sublinear extending down to clumps with $L_{\mathrm{TIR}} \sim 10^{3} L_{\odot}$. These correlations become linear when extended to external galaxies. A bimodal behavior in the $L_{\mathrm{TIR}}-L_{\mathrm{mol}}^{\prime}$ correlations was found for clumps with different dust temperature, luminosity-to-mass ratio, and $\sigma_{\text {line }} / \sigma_{\text {vir }}$. Such bimodal behavior may be due to evolutionary effects. The slopes of $L_{\mathrm{TIR}}-L_{\text {mol }}^{\prime}$ correlations become more shallow as clumps evolve. We compared our results with lower $J$ transition lines in Wu et al. (2010). The correlations between clump masses and line luminosities are close to linear for low effective excitation density tracers but become sublinear for high effective excitation density tracers for clumps with $L_{\mathrm{TIR}}$ larger than $L_{\mathrm{TIR}} \sim 10^{4.5} L_{\odot}$. High effective excitation density tracers cannot linearly trace the total clump masses, leading to a sublinear correlations for both $M_{\text {clump }}-L_{\text {mol }}^{\prime}$ and $L_{\mathrm{TIR}}{ }^{-}$ $L_{\text {mol }}^{\prime}$ relations.
\end{abstract}

Key words: galaxies: evolution - galaxies: ISM - ISM: kinematics and dynamics - stars: formation

\section{INTRODUCTION}

The empirical correlation between the star formation rate (SFR) surface density $\left(\Sigma_{\mathrm{SFR}}\right)$ and the surface density of cold gas $\left(\Sigma_{\text {gas }}\right)$ pioneered in the works of Schmidt (1959) and Kennicutt (1998), the so-called Kennicutt-Schmidt (K-S) law, is of great importance for input into theoretical models of galaxy evolution. The power-law relations between $\Sigma_{\mathrm{SFR}}$ and total gas surface density $\left(\Sigma_{\text {gas }}=\Sigma_{\mathrm{H}_{\mathrm{I}}}+\Sigma_{\mathrm{H}_{2}}\right)$ used to describe star formation across entire galaxies and galactic nuclei, have a typical power-law index of 1.4-1.6 (Kennicutt 1998; Kennicutt \& Evans 2012).

However, since stars form in dense cores of molecular clouds, the relations between $\Sigma_{\mathrm{SFR}}$ and total molecular gas surface density $\left(\Sigma_{\mathrm{mol}}\right)$ may be more fundamental to describe star formation. Indeed, very tight relations between SFR indicated by infrared luminosities $\left(L_{\mathrm{IR}}\right)$ and dense molecular gas mass indicated by molecular line luminosities $\left(L_{\text {mol }}^{\prime}\right)$ have been revealed in external galaxies (Gao \& Solomon 2004; Greve et al. 2014; Zhang et al. 2014; Liu et al. 2015).

Interestingly, the $L_{\mathrm{IR}}-L_{\mathrm{mol}}^{\prime}$ relations are close to linear for $J=1-0$ up to $J=5-4$ of $\mathrm{CO}$ (Greve et al. 2014). From $J=6-5$ and up to the $J=13-12$ transition of CO, Greve et al. (2014) found an increasingly sublinear slope with increasing $J$. They argued that the thermal state of high $J$ CO transitions is unlikely to be maintained by star formation-powered far-UV radiation fields and thus is no longer directly tied to the SFR. Such trend is similar to the predictions in simulations (Narayanan et al. 2008).

However, from Herschel SPIRE FTS observations of 167 local galaxies, Liu et al. (2015) found that the $L_{\mathrm{IR}}-L_{\text {mol }}^{\prime}$ relations are essentially linear and tight for all the $\mathrm{CO}$ transitions including the highest $J=12-11$ transition, indicating that the SFR is linearly correlated with the dense molecular 
gas. Liu et al. (2015) argued that the nonlinear result reported in Greve et al. (2014) can be attributed to the comparatively small number of galaxies in their sample.

Similarly, nearly linear $L_{\mathrm{IR}}-L_{\text {mol }}^{\prime}$ correlations for dense molecular gas tracers (e.g., HCN and CS) were found toward both Galactic dense clumps and galaxies (Gao \& Solomon 2004; Wu et al. 2005, 2010; Ma et al. 2013; Zhang et al. 2014), indicating a constant SFR per unit mass from the scale of dense clumps to that of distant galaxies (Wu et al. 2005, 2010). And dense molecular line luminosity seem to be linearly related to the mass of dense gas most relevant to star formation ( $\mathrm{Wu}$ et al. 2010; Reiter et al. 2011). Through observations of nearby clouds, Lada et al. (2010) found that SFR in molecular clouds seems to be linearly proportional to the cloud mass above an extinction threshold of $A_{K} \approx 0.8 \mathrm{mag}$. If star formation only depends on the mass of dense gas, the underlying star formation scaling law will be always linear for clouds and galaxies with the same dense gas fraction (Lada et al. 2012).

However, apparently sublinear $L_{\mathrm{IR}}-L_{\text {mol }}^{\prime}$ correlations were found for middle $J$ dense line tracers like HCN (3-2) and CS (76) in Galactic clumps (Wu et al. 2010). In contrast, linear $L_{\mathrm{IR}}{ }^{-}$ $L_{\text {mol }}^{\prime}$ correlations for $J=7-6$ of CS, $J=4-3$ of $\mathrm{HCN}$ and $\mathrm{HCO}^{+}$were found in nearby star-forming galaxies (SFGs) (Zhang et al. 2014). Are the linear observed K-S scaling relations for dense gas in galaxies an artifact of unresolved measurements of GMCs due to the beam dilution as suggested by Lada et al. (2013)?

In spite of dense molecular gas tracers, optically thin dust continuum emission at (sub)millimeter wavelengths has often been used as a tracer for molecular gas masses in extragalactic studies (Dunne et al. 2000; Scoville et al. 2016). Dunne et al. (2000) compared the dust masses, derived from the submillimetre fluxes, to the $\mathrm{H}_{2}, \mathrm{HI}$ and $\mathrm{H}_{2}+\mathrm{H}$ I masses in 104 Local universe galaxies. The strongest correlation is with the molecular gas, which suggests that the dust is primarily found in molecular clouds (Dunne et al. 2000). Strong correlations between dust masses and SFRs have been revealed in both low $z$ and high $z$ galaxies (Dunne et al. 2000; Clements et al. 2010; da Cunha et al. 2010; Rowlands et al. 2012; Clemens et al. 2013; Magdis et al. 2013; Hjorth et al. 2014; Santini et al. 2014; Scoville et al. 2016). In this work, we will also explore the star formation law in Galactic massive clumps with dust continuum emission and compare with extragalactic studies.

\section{OBSERVATIONS}

\subsection{The Sample}

The sample of 146 sources in the survey was obtained from Faúndez et al. (2004). The basic parameters (like coordinates, sizes, masses, temperature, bolometric luminosities, densities) of these sources were summarized in Table 1 of Faúndez et al. (2004). The 146 sources are IRAS point sources and the main clumps in $1.2 \mathrm{~mm}$ continuum emission, which have far-infrared colors typical of ultra-compact H II (UC H II) regions. Most of the sources $(91 \%)$ have bolometric luminosities $\geqslant 10^{4} L_{\odot}$, indicating that they harbor at least one B0.5 type massive star (Faúndez et al. 2004). The median and maximum bolometric luminosities of this sample are $6.4 \times 10^{4}$ and $5.6 \times 10^{6} L_{\odot}$, respectively. The dust temperature ranges from 18 to $46 \mathrm{~K}$ with a median value of $31 \mathrm{~K}$ (Faúndez et al. 2004). The volume densities of these sources, which were derived assuming that the clumps have spherical morphologies, range from $5.5 \times 10^{3}$ to $3.8 \times 10^{6}$ with a median value of $\sim 1 \times 10^{5} \mathrm{~cm}^{-3}$, spanning three orders of magnitude (Faúndez et al. 2004). Considering the large diversity of both densities and bolometric luminosities, this sample is ideal for studies of the K-S law in the Milky Way.

\subsection{Observations with the ASTE $10 \mathrm{~m}$ Telescope}

The single pointing observations toward the 146 Galactic clumps were conducted with the Atacama Submillimeter Telescope Experiment (ASTE) $10 \mathrm{~m}$ telescope with position switching mode between 2014 July 1st and 4th. The pointing positions were presented in Table 1 of Faúndez et al. (2004). The two-sideband single-polarization heterodyne receiver DASH345/CATS345, operating at frequencies of 324-372 GHz was used to observe HCN (4-3) and CS (7-6) lines simultaneously. We used the MAC spectrometer with a spectral resolution of $0.5 \mathrm{MHz}$ or $0.42 \mathrm{~km} \mathrm{~s}^{-1}$. The beam size at $354 \mathrm{GHz}$ is $\sim 22^{\prime \prime}$. The main beam efficiency was $\sim 0.6$. The system temperature varied from 400 to $500 \mathrm{~K}$ during observations. The rms level per channel is $\sim 0.1-0.2 \mathrm{~K}$ in antenna temperature. The data were reduced with Gildas/Class package (Guilloteau \& Lucas 2000). Baseline subtraction was performed by fitting linear functions.

\section{RESULTS}

\subsection{Observational Results of Molecular Lines}

We detected HCN (4-3) toward 141 clumps and CS (7-6) toward 137 clumps. In Figure 1, we show the HCN (4-3) and CS (7-6) spectra of two sources for example. For IRAS 183170757, both HCN (4-3) and CS (7-6) spectra can be well fitted with single Gaussian profiles. For IRAS 09018-4816, CS (7-6) has a Gaussian profile, while HCN (4-3) shows asymmetric profiles with an absorption dip. There are $74(\sim 52 \%)$ clumps showing asymmetric profiles in HCN (4-3) like IRAS 090184816. While there are only $22(16 \%)$ clumps showing asymmetric profiles in CS (7-6). The line profiles were classified into three categories as shown in the 6th and 11th columns in Table 1. Those denoted with "G" have symmetric profiles which can be well fitted with Gaussian profiles. Those denoted with "B" or "R" have asymmetric profiles with their observed emission peaks blueshifted or redshifted with respect to the peak velocities derived from Gaussian fits, respectively. In this work, we will not discuss the line profiles in details. The line profiles will be modeled and discussed in another paper for kinematics (e.g., infall and outflow) studies.

Many lines especially HCN (4-3) lines with asymmetric profiles show absorption dips due to self-absorption. Previous works like Wu et al. $(2005,2010)$ and Stephens et al. (2016) simply used Moment 0 method to calculating the integrated intensity of such optically thick line, which may severely underestimate the total emission including the blocked radiation due to self-absorption (van Kempen et al. 2009; Ossenkopf et al. 2010). Gaussian fits were usually performed to obtain a rough correction for self-absorption and to compare with radiation transfer models (van Kempen et al. 2009; Ossenkopf et al. 2010). In this work, we fitted all the detected spectra with Gaussian functions and present the results (integrated intensity $\int T_{\mathrm{A}}^{*} d V$, systemic velocity $V_{\mathrm{ls}}$, the FWHM and peak antenna temperature $T_{\mathrm{A}}^{*}$ ) in Table 1 . Due to selfabsorption, most HCN (4-3) lines have larger errors in the 


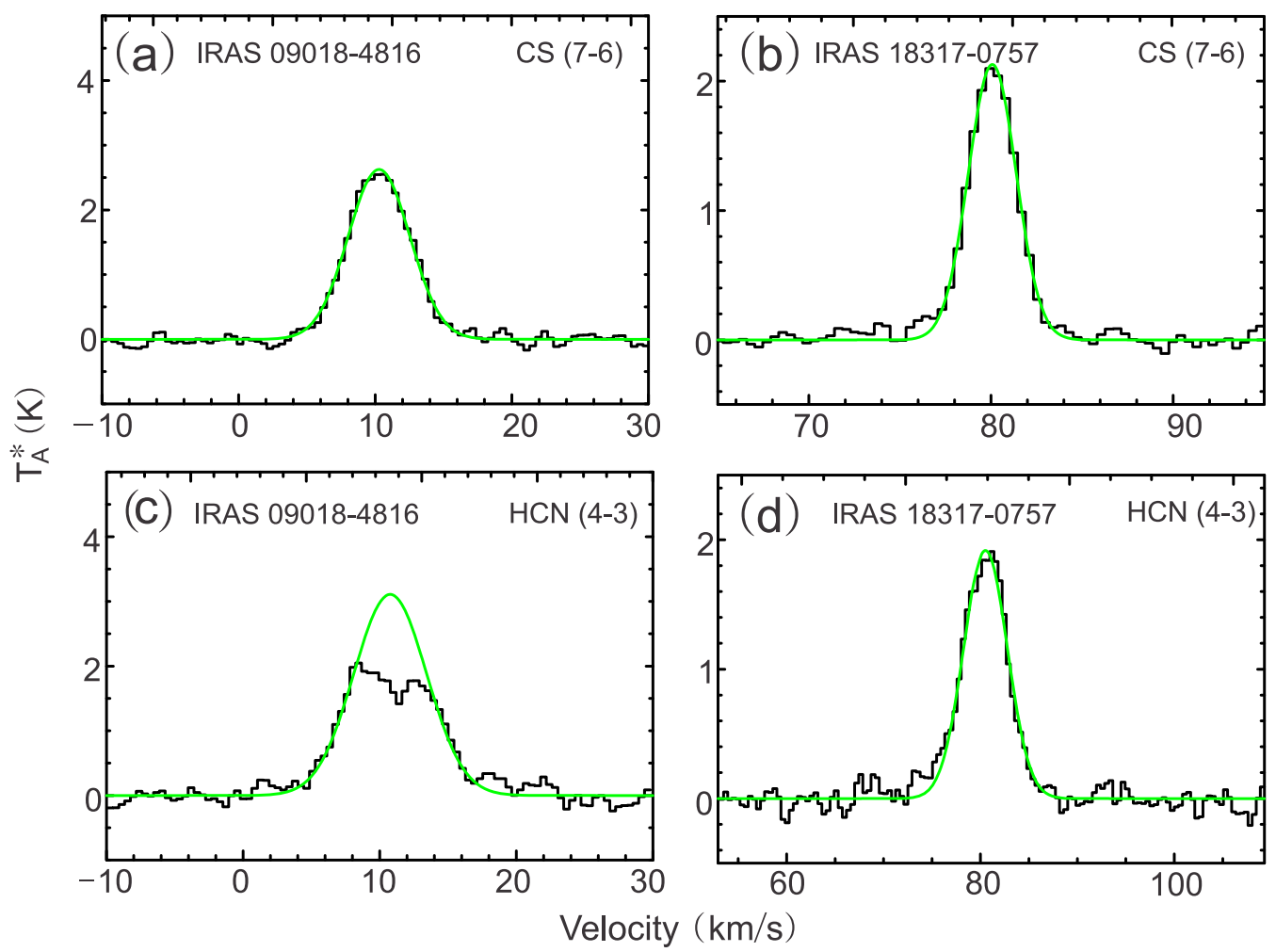

Figure 1. Spectra of IRAS 09018-4816 (left panels) and IRAS 18317-0757 (right panels). The upper panels show CS (7-6) lines. The lower panels show HCN (4-3) lines. The green lines are Gaussian fits.

integrated intensities from Gaussian fit than CS (7-6) lines. In Figure 2, we compare the integrated intensities inferred from Gaussian fits with the values simply obtained from integrating the lines (Moment 0). The integrated intensities obtained from different methods are very consistent and roughly linearly correlated. Therefore, we claim that the integrated intensities obtained from Gaussian fits are reliable.

The median line widths for HCN (4-3) lines with symmetric profiles and with asymmetric profiles are $\sim 5.3$ and $\sim 8.1 \mathrm{~km} \mathrm{~s}^{-1}$, respectively. The median line widths for CS (76) lines with symmetric profiles and with asymmetric profiles are $\sim 4.0$ and $\sim 7.5 \mathrm{~km} \mathrm{~s}^{-1}$, respectively. In general, lines with asymmetric profiles have much larger line widths than lines with symmetric profiles, indicating that those clumps with asymmetric line profiles may be more turbulent and more likely be affected by stellar feedback or bulk motions (e.g., infall, outflows). CS (7-6) lines usually have smaller line widths than $\mathrm{HCN}$ (4-3). The median line width ratio of CS (7-6) to HCN (43 ) is $\sim 0.67$.

\subsection{Line Luminosities}

Assuming a Gaussian brightness distribution for the source and a Gaussian beam, molecular line luminosities $L_{\text {mol }}^{\prime}$ can be derived following $\mathrm{Wu}$ et al. (2010)

$$
\begin{aligned}
L_{\text {mol }}^{\prime}= & 23.5 \times 10^{-6} \times D^{2} \times\left(\frac{\pi \times \theta_{\mathrm{s}}^{2}}{4 \ln 2}\right) \times\left(\frac{\theta_{\mathrm{s}}^{2}+\theta_{\text {beam }}^{2}}{\theta_{\mathrm{s}}^{2}}\right) \\
& \times \int T_{\mathrm{A}}^{*} d V / \eta .
\end{aligned}
$$

Here $D$ is the distance in kpc obtained from Faúndez et al. (2004), and $\theta_{\mathrm{s}}$ and $\theta_{\text {beam }}$ are the size of the line emission source and of the beam in arcsecond, and $\eta$ is the main beam efficiency. Since we only carried out single pointing observations and thus can not determine the exact line emission area, we here assume that the source sizes of HCN (4-3) and CS (76) are the same as that of $1.2 \mathrm{~mm}$ continuum emission in Faúndez et al. (2004). The caveats in estimating line luminosity will be discussed in Section 4.1.

The derived $\mathrm{HCN}$ (4-3) ( $\left.L_{\mathrm{HCN}}^{\prime}\right)$ and $\mathrm{CS}$ (7-6) $\left(L_{\mathrm{CS}}^{\prime}\right)$ luminosities are listed in the 4th and 5th columns of Table 2. The median values of $L_{\mathrm{HCN}}^{\prime}$ and $L_{\mathrm{CS}}^{\prime}$ are $\sim 16.4$ and $\sim 9.2 \mathrm{~K} \mathrm{~km} \mathrm{~s}^{-1} \mathrm{pc}^{2}$, respectively. The mean values of $L_{\mathrm{HCN}}^{\prime}$ and $L_{\mathrm{CS}}^{\prime}$ are $\sim 62.5$ and $\sim 56.6 \mathrm{~K} \mathrm{~km} \mathrm{~s}^{-1} \mathrm{pc}^{2}$, respectively.

\subsection{Gravitational Stability of Dense Clumps}

To examine the gravitational stability of those dense clumps, we derive one-dimensional virial velocity dispersions $\left(\sigma_{\mathrm{vir}}\right)$ :

$$
\sigma_{\text {vir }}=\sqrt{\frac{\gamma M_{\text {clump }} G}{5 R_{\text {clump }}}} .
$$

Here $M_{\text {clump }}$ is the clump mass, $G$ is the gravitational constant, and $R_{\text {clump }}$ is the radius of the dense clump. $M_{\text {clump }}$ and $R_{\text {clump }}$ were taken from Faúndez et al. (2004). Parameter $\gamma$ is the geometric factor equal to unity for a uniform density profile and $5 / 3$ for an inverse square profile (Williams et al. 1994). Here we take $\gamma$ equal to $5 / 3$. The derived one-dimensional virial velocity dispersions $\left(\sigma_{\text {vir }}\right)$ are listed in the 6th column of Table 2. 
Table 1

Parameters of Molecular Lines in Single-dish Observations

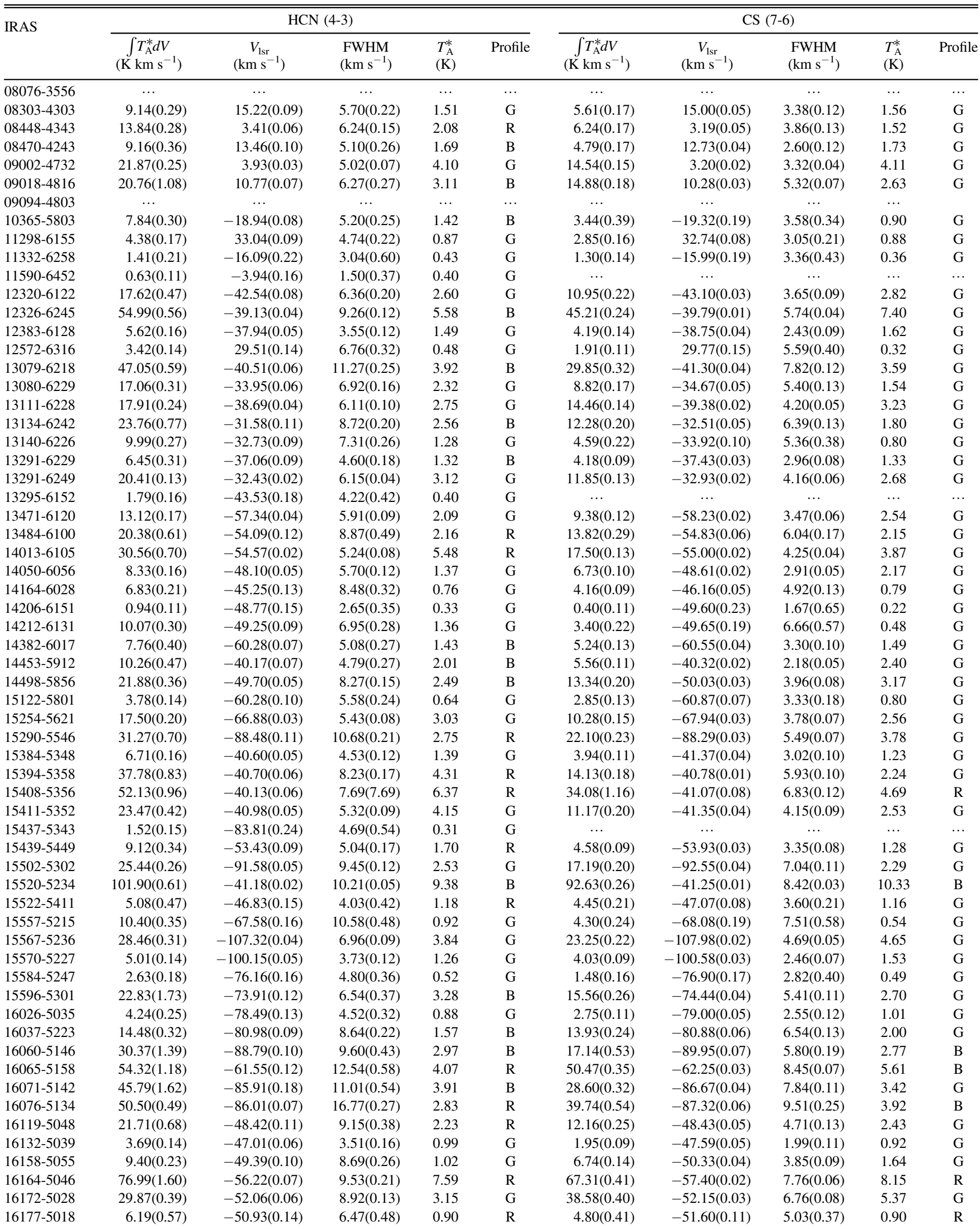


Table 1

(Continued)

\begin{tabular}{|c|c|c|c|c|c|c|c|c|c|c|}
\hline \multirow{2}{*}{ IRAS } & \multicolumn{5}{|c|}{$\mathrm{HCN}(4-3)$} & \multicolumn{5}{|c|}{ CS (7-6) } \\
\hline & $\begin{array}{c}\int_{(\mathrm{K} \mathrm{km} \mathrm{s}} T_{\mathrm{A}}^{*} d V \\
\text {.1 }\end{array}$ & $\begin{array}{c}V_{\mathrm{lsr}} \\
\left(\mathrm{km} \mathrm{s}^{-1}\right)\end{array}$ & $\begin{array}{l}\text { FWHM } \\
\left(\mathrm{km} \mathrm{s}^{-1}\right)\end{array}$ & $\begin{array}{l}T_{\mathrm{A}}^{*} \\
(\mathrm{~K})\end{array}$ & Profile & $\begin{array}{c}\int_{(\mathrm{K} \mathrm{km} \mathrm{s}} T_{\mathrm{A}}^{*} d V \\
\text {.1 }\end{array}$ & $\begin{array}{c}V_{\mathrm{lsr}} \\
\left(\mathrm{km} \mathrm{s}^{-1}\right)\end{array}$ & $\begin{array}{l}\text { FWHM } \\
\left(\mathrm{km} \mathrm{s}^{-1}\right)\end{array}$ & $\begin{array}{l}T_{\mathrm{A}}^{*} \\
(\mathrm{~K})\end{array}$ & Profile \\
\hline $16272-4837$ & $30.11(0.83)$ & $-45.79(0.11)$ & $9.06(0.72)$ & 3.12 & B & $12.29(0.20)$ & $-46.42(0.04)$ & $5.81(0.12)$ & 1.99 & $\mathrm{G}$ \\
\hline $16297-4757$ & $10.53(0.24)$ & $-79.92(0.08)$ & $6.89(0.19)$ & 1.44 & $\mathrm{G}$ & $12.69(0.16)$ & $-79.72(0.03)$ & $5.68(0.09)$ & 2.10 & $\mathrm{G}$ \\
\hline $16304-4710$ & $1.81(0.12)$ & $-61.00(0.13)$ & $3.87(0.28)$ & 0.44 & G & $0.76(0.11)$ & $-62.28(0.24)$ & $3.56(0.63)$ & 0.20 & $\mathrm{G}$ \\
\hline $16313-4729$ & $5.86(0.16)$ & $-72.29(0.08)$ & $6.09(0.20)$ & 0.90 & $\mathrm{G}$ & $3.00(0.16)$ & $-73.20(0.18)$ & $7.06(0.46)$ & 0.40 & $\mathrm{G}$ \\
\hline $16318-4724$ & $15.54(0.39)$ & $-120.54(0.09)$ & $9.10(0.30)$ & 1.60 & $\mathrm{R}$ & 11.67(0.17) & $-121.43(0.05)$ & $7.27(0.13)$ & 1.51 & $\mathrm{G}$ \\
\hline $16330-4725$ & $19.56(0.17)$ & $-74.09(0.03)$ & $6.99(0.07)$ & 2.63 & G & $14.60(0.13)$ & $-74.62(0.02)$ & $5.70(0.06)$ & 2.41 & $\mathrm{G}$ \\
\hline $16344-4658$ & $16.36(0.39)$ & $-48.85(0.10)$ & $12.41(0.30)$ & 1.24 & B & $12.84(0.54)$ & $-48.96(0.07)$ & $7.75(0.23)$ & 1.56 & B \\
\hline $16348-4654$ & $31.52(0.50)$ & $-47.23(0.08)$ & $12.21(0.23)$ & 2.43 & B & $17.65(0.21)$ & $-47.92(0.04)$ & $7.32(0.11)$ & 2.27 & $\mathrm{G}$ \\
\hline $16351-4722$ & $61.00(1.15)$ & $-40.30(0.06)$ & $9.10(0.20)$ & 6.29 & B & $55.22(0.67)$ & $-40.64(0.04)$ & $7.22(0.10)$ & 7.18 & B \\
\hline $16362-4639$ & $2.68(0.13)$ & $-37.38(0.12)$ & $5.07(0.31)$ & 0.50 & $\mathrm{G}$ & $0.53(0.07)$ & $-37.93(0.12)$ & $1.85(0.30)$ & 0.27 & $\mathrm{G}$ \\
\hline $16372-4545$ & $10.64(0.33)$ & $-57.54(0.06)$ & $5.69(0.18)$ & 1.76 & $\mathrm{R}$ & $8.72(0.26)$ & $-58.44(0.06)$ & $4.48(0.11)$ & 1.83 & $\mathrm{R}$ \\
\hline $16385-4619$ & $21.37(0.30)$ & $-117.85(0.07)$ & $10.59(0.26)$ & 1.90 & B & $19.40(0.16)$ & $-118.02(0.03)$ & $6.45(0.07)$ & 2.82 & $\mathrm{G}$ \\
\hline $16424-4531$ & $17.39(0.30)$ & $-31.06(0.05)$ & $7.89(0.19)$ & 2.07 & $\mathrm{G}$ & $3.58(0.18)$ & $-34.57(0.07)$ & $2.96(0.20)$ & 1.13 & $\mathrm{G}$ \\
\hline $16445-4459$ & $10.37(0.23)$ & $-121.43(0.07)$ & $6.92(0.22)$ & 1.41 & $\mathrm{G}$ & $4.55(0.15)$ & $-122.31(0.08)$ & $5.42(0.24)$ & 0.79 & G \\
\hline $16458-4512$ & $17.40(0.36)$ & $-49.92(0.07)$ & $7.22(0.24)$ & 2.27 & $\mathrm{R}$ & $4.57(0.24)$ & $-50.75(0.09)$ & $4.18(0.29)$ & 1.03 & G \\
\hline $16484-4603$ & $25.05(0.62)$ & $-31.58(0.08)$ & 7.53(0.32) & 3.13 & $\mathrm{R}$ & $16.30(0.23)$ & $-32.19(0.03)$ & $4.76(0.11)$ & 3.21 & $\mathrm{G}$ \\
\hline $16487-4423$ & $6.76(0.17)$ & $-43.24(0.06)$ & $5.75(0.19)$ & 1.11 & $\mathrm{R}$ & $2.35(0.11)$ & $-43.92(0.08)$ & $3.45(0.19)$ & 0.64 & $\mathrm{G}$ \\
\hline $16489-4431$ & $6.20(0.18)$ & $-40.40(0.07)$ & $5.06(0.19)$ & 1.15 & $\mathrm{G}$ & $2.75(0.11)$ & $-40.74(0.06)$ & $3.18(0.16)$ & 0.81 & $\mathrm{G}$ \\
\hline $16506-4512$ & $19.81(0.39)$ & $-25.80(0.03)$ & $4.31(0.07)$ & 4.31 & $\mathrm{R}$ & $12.18(0.21)$ & $-25.84(0.02)$ & $2.84(0.04)$ & 4.02 & $\mathrm{R}$ \\
\hline $16524-4300$ & $19.11(0.38)$ & $-40.56(0.05)$ & $5.38(0.07)$ & 3.34 & $\mathrm{R}$ & $9.87(0.11)$ & $-41.30(0.02)$ & $3.49(0.05)$ & 2.66 & $\mathrm{G}$ \\
\hline $16547-4247$ & $117.07(1.30)$ & $-29.73(0.08)$ & $17.16(0.31)$ & 6.41 & $\mathrm{~B}$ & $53.42(0.37)$ & $-30.27(0.03)$ & $9.95(0.11)$ & 5.05 & B \\
\hline $16562-3959$ & $71.50(0.48)$ & $-11.90(0.02)$ & $6.85(0.06)$ & 9.80 & $\mathrm{R}$ & $38.38(0.22)$ & $-12.53(0.01)$ & $4.55(0.03)$ & 7.93 & $\mathrm{G}$ \\
\hline $16571-4029$ & $20.79(1.13)$ & $-14.69(0.12)$ & $5.65(0.41)$ & 3.46 & $\mathrm{R}$ & $14.24(0.27)$ & $-15.03(0.03)$ & $3.12(0.07)$ & 4.29 & $\mathrm{G}$ \\
\hline $17006-4215$ & $15.92(0.26)$ & $-24.22(0.05)$ & $5.71(0.11)$ & 2.62 & $\mathrm{G}$ & $13.40(0.13)$ & $-24.69(0.01)$ & $3.06(0.04)$ & 4.12 & $\mathrm{G}$ \\
\hline $17008-4040$ & $32.02(0.67)$ & $-15.93(0.07)$ & $6.67(0.12)$ & 4.51 & $\mathrm{R}$ & $20.00(0.24)$ & $-16.67(0.03)$ & $5.01(0.07)$ & 3.75 & $\mathrm{G}$ \\
\hline $17016-4124$ & $83.23(1.44)$ & $-26.48(0.11)$ & $12.89(0.45)$ & 6.07 & $\mathrm{R}$ & $32.83(0.42)$ & $-26.80(0.05)$ & $9.12(0.15)$ & 3.38 & $\mathrm{G}$ \\
\hline 17136-3617 & 27.31(0.16) & $-10.27(0.01)$ & 4.99(0.04) & 5.14 & $\mathrm{G}$ & 20.77(0.11) & $-10.99(0.01)$ & $3.47(0.02)$ & 5.62 & $\mathrm{G}$ \\
\hline $17143-3700$ & $11.09(0.26)$ & $-31.32(0.07)$ & 7.94(0.24) & 1.31 & $\mathrm{~B}$ & 7.38(0.13) & $-31.81(0.04)$ & $4.56(0.10)$ & 1.52 & $\mathrm{G}$ \\
\hline $17158-3901$ & $35.28(0.66)$ & $-15.85(0.07)$ & $10.25(0.29)$ & 3.23 & $\mathrm{R}$ & $20.34(0.14)$ & $-16.19(0.00)$ & $6.08(0.05)$ & 3.14 & $\mathrm{G}$ \\
\hline $17160-3707$ & $18.45(0.43)$ & $-69.65(0.06)$ & $7.47(0.19)$ & 2.32 & B & $10.52(0.14)$ & $-69.85(0.03)$ & $4.53(0.07)$ & 2.18 & $\mathrm{G}$ \\
\hline $17175-3544$ & $91.17(1.20)$ & $-5.74(0.06)$ & $11.00(0.25)$ & 7.79 & $\mathrm{R}$ & $84.85(0.19)$ & $-6.54(0.01)$ & $6.55(0.02)$ & 12.17 & $\mathrm{G}$ \\
\hline $17204-3636$ & $14.48(0.82)$ & $-17.76(0.10)$ & $5.66(0.45)$ & 2.40 & B & $6.05(0.16)$ & $-17.94(0.05)$ & $3.66(0.12)$ & 1.55 & $\mathrm{G}$ \\
\hline 17220-3609 & $41.77(0.40)$ & $-95.04(0.06)$ & $12.73(0.23)$ & 3.08 & B & $35.20(0.16)$ & $-94.67(0.02)$ & $6.65(0.04)$ & 4.97 & $\mathrm{G}$ \\
\hline $17233-3606$ & $182.37(0.81)$ & $-4.15(0.04)$ & $21.04(0.15)$ & 8.14 & $\mathrm{R}$ & $113.92(0.84)$ & $-3.16(0.03)$ & $10.33(0.14)$ & 10.36 & $\mathrm{R}$ \\
\hline $17244-3536$ & $5.39(0.12)$ & $-9.36(0.04)$ & $3.87(0.10)$ & 1.31 & $\mathrm{G}$ & $2.63(0.10)$ & $-10.50(0.05)$ & $2.84(0.13)$ & 0.87 & $\mathrm{G}$ \\
\hline $17258-3637$ & $46.49(1.51)$ & $-11.68(0.06)$ & 7.64(0.19) & 5.72 & $\mathrm{R}$ & $35.66(0.29)$ & $-12.11(0.02)$ & $5.38(0.04)$ & 6.22 & $\mathrm{R}$ \\
\hline 17269-3312 & $3.36(0.22)$ & $-20.70(0.20)$ & $5.57(0.82)$ & 0.57 & G & $0.69(0.08)$ & $-21.60(0.17)$ & $3.06(0.40)$ & 0.21 & $\mathrm{G}$ \\
\hline $17271-3439$ & $30.21(2.53)$ & $-15.86(0.43)$ & $10.27(3.36)$ & 2.76 & B & $24.24(0.33)$ & $-15.40(0.03)$ & $4.55(0.07)$ & 5.01 & $\mathrm{G}$ \\
\hline $17278-3541$ & $7.40(0.23)$ & $0.73(0.07)$ & $4.71(0.21)$ & 1.48 & G & $3.19(0.14)$ & $0.35(0.08)$ & $3.58(0.15)$ & 0.84 & $\mathrm{G}$ \\
\hline $17439-2845$ & $4.54(0.15)$ & $18.76(0.07)$ & $4.32(0.17)$ & 0.99 & G & $1.56(0.13)$ & $18.15(0.11)$ & $3.90(0.55)$ & 0.38 & $\mathrm{G}$ \\
\hline $17441-2822$ & $186.66(40.2)$ & $51.49(1.53)$ & $15.12(1.81)$ & 11.60 & B & $262.61(1.23)$ & $60.05(0.03)$ & $22.95(0.10)$ & 10.75 & B \\
\hline $17455-2800$ & $17.20(0.15)$ & $-14.19(0.02)$ & $5.62(0.06)$ & 2.88 & G & $10.28(0.13)$ & $-14.79(0.02)$ & $3.87(0.06)$ & 2.50 & $\mathrm{G}$ \\
\hline $17545-2357$ & $5.85(0.11)$ & $9.16(0.04)$ & $3.98(0.09)$ & 1.38 & $\mathrm{G}$ & $2.58(0.08)$ & $8.57(0.04)$ & $2.99(0.10)$ & 0.81 & $\mathrm{G}$ \\
\hline $17589-2312$ & $6.92(0.49)$ & $21.64(0.18)$ & $6.07(0.25)$ & 1.07 & $\mathrm{R}$ & $1.79(0.07)$ & 20.81(0.08) & $4.05(0.17)$ & 0.42 & $\mathrm{G}$ \\
\hline $17599-2148$ & $12.69(0.42)$ & $19.75(0.06)$ & $5.34(0.12)$ & 2.23 & $\mathrm{R}$ & $6.28(0.09)$ & $19.16(0.04)$ & $4.79(0.08)$ & 1.23 & G \\
\hline $18032-2032$ & $47.73(0.78)$ & $5.86(0.10)$ & $12.55(0.26)$ & 3.57 & $\mathrm{R}$ & $32.22(0.46)$ & $4.49(0.05)$ & $8.00(0.17)$ & 3.78 & $\mathrm{R}$ \\
\hline 18056-1952 & $51.66(1.12)$ & $66.70(0.10)$ & $11.03(0.33)$ & 4.40 & B & $26.98(0.43)$ & $66.75(0.07)$ & $9.34(0.17)$ & 2.71 & B \\
\hline $18075-2040$ & $\ldots$ & $\ldots$ & .. & $\ldots$ & $\ldots$ & $\ldots$ & $\ldots$ & .. & $\ldots$ & $\ldots$ \\
\hline $18079-1756$ & $14.09(0.16)$ & $18.39(0.02)$ & $4.58(0.08)$ & 2.89 & G & $8.67(0.10)$ & $17.68(0.01)$ & $2.70(0.04)$ & 3.02 & $\mathrm{G}$ \\
\hline 18089-1732 & $26.06(0.96)$ & $33.52(0.06)$ & $6.39(0.23)$ & 3.83 & B & $16.48(0.14)$ & $33.13(0.02)$ & $4.65(0.06)$ & 3.33 & B \\
\hline 18110-1854 & $18.81(0.29)$ & $39.04(0.03)$ & $5.90(0.14)$ & 2.99 & $\mathrm{R}$ & $8.88(0.08)$ & $38.55(0.02)$ & $3.72(0.04)$ & 2.24 & $\mathrm{G}$ \\
\hline 18116-1646 & $23.90(0.53)$ & $50.25(0.03)$ & $6.71(0.12)$ & 3.35 & B & $21.86(1.22)$ & 49.47(0.06) & $4.02(0.13)$ & 5.11 & B \\
\hline $18117-1753$ & $34.21(0.87)$ & $38.00(0.09)$ & $9.14(0.29)$ & 3.52 & $\mathrm{R}$ & $20.85(0.28)$ & $36.46(0.04)$ & $6.47(0.06)$ & 3.03 & $\mathrm{G}$ \\
\hline 18134-1942 & $10.81(0.12)$ & $10.74(0.02)$ & $3.50(0.05)$ & 2.90 & $\mathrm{G}$ & $5.15(0.07)$ & $10.14(0.02)$ & $2.44(0.04)$ & 1.99 & $\mathrm{G}$ \\
\hline $18139-1842$ & $13.05(0.84)$ & $39.98(0.05)$ & $4.44(0.21)$ & 2.76 & $\mathrm{R}$ & 7.44(0.12) & $39.61(0.03)$ & $3.39(0.06)$ & 2.06 & $\mathrm{G}$ \\
\hline 18159-1648 & $39.77(0.77)$ & $23.88(0.07)$ & $7.66(0.26)$ & 4.88 & $\mathrm{R}$ & $28.10(0.13)$ & $22.38(0.01)$ & $5.42(0.03)$ & 4.87 & G \\
\hline $18182-1433$ & $14.95(0.48)$ & $60.11(0.10)$ & $7.39(0.29)$ & 1.90 & B & $6.99(0.25)$ & $59.51(0.10)$ & $5.47(0.18)$ & 1.20 & $\mathrm{G}$ \\
\hline $18223-1243$ & $3.85(0.14)$ & $45.97(0.07)$ & $4.29(0.19)$ & 0.84 & $\mathrm{G}$ & $1.67(0.09)$ & $45.15(0.06)$ & $2.38(0.18)$ & 0.66 & $\mathrm{G}$ \\
\hline $18228-1312$ & $11.00(0.42)$ & $33.64(0.08)$ & $5.44(0.16)$ & 1.90 & B & $4.62(0.33)$ & $33.69(0.12)$ & $3.86(0.17)$ & 1.13 & $\mathrm{R}$ \\
\hline $18236-1205$ & $17.34(0.47)$ & $27.81(0.10)$ & $8.51(0.47)$ & 1.92 & $\mathrm{R}$ & $6.17(0.25)$ & $26.81(0.09)$ & $5.37(0.29)$ & 1.08 & $\mathrm{G}$ \\
\hline $18264-1152$ & $32.26(0.59)$ & $45.38(0.07)$ & $8.46(0.20)$ & 3.58 & $\mathrm{G}$ & $12.06(0.21)$ & $43.95(0.03)$ & $4.49(0.10)$ & 2.52 & G \\
\hline 18290-0924 & $\ldots$ & $\ldots$ & $\ldots$ & $\ldots$ & $\cdots$ & $\ldots$ & $\ldots$ & $\ldots$ & $\ldots$ & $\cdots$ \\
\hline
\end{tabular}


Table 1

(Continued)

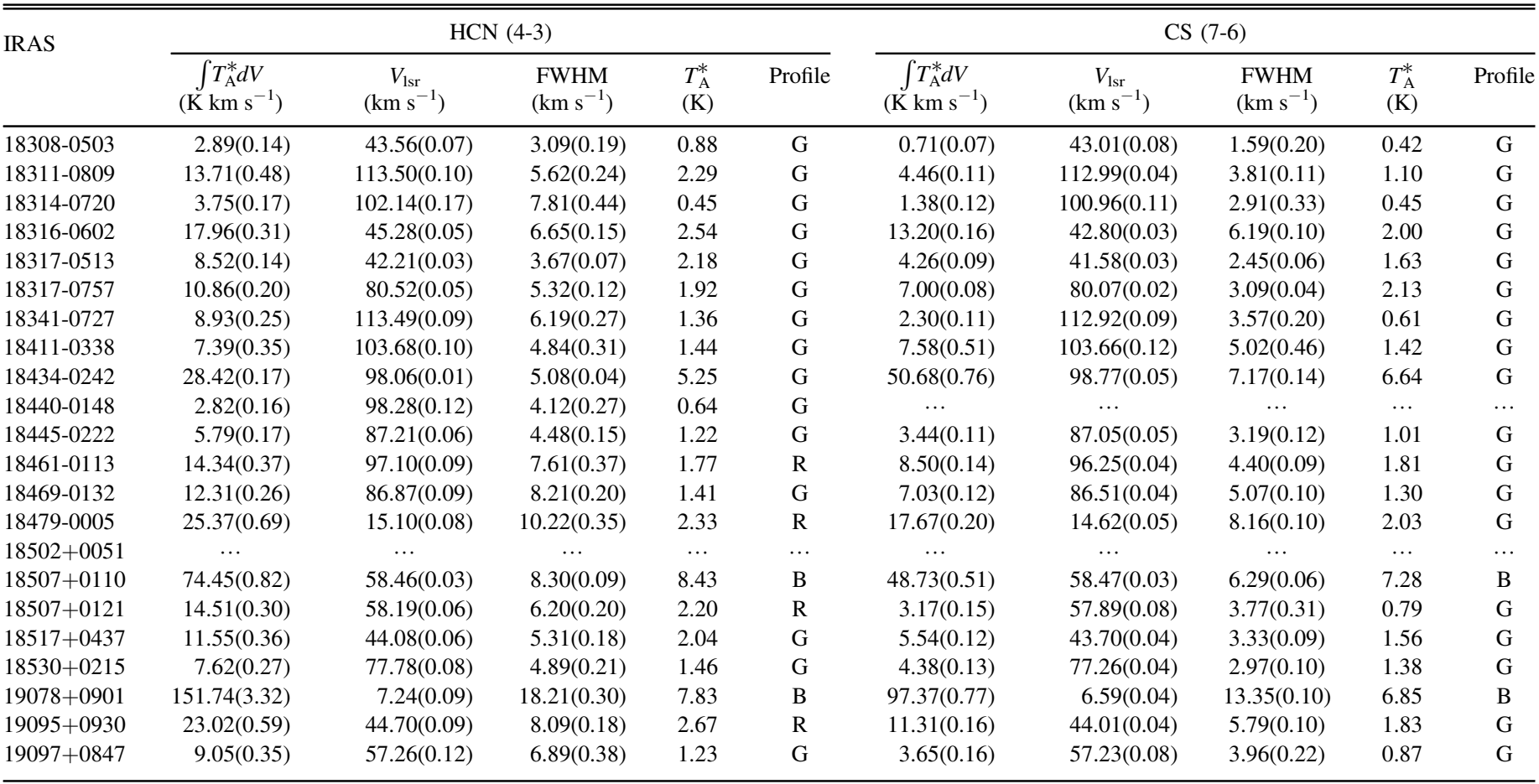

Assuming the line profiles are Gaussian, the one dimensional velocity dispersions of molecular lines are

$$
\sigma_{\text {line }}=\frac{\text { FWHM }}{2 \sqrt{2 \ln (2)}}=\frac{\text { FWHM }}{2.355} .
$$

We derived $\sigma_{\mathrm{HCN}}$ and $\sigma_{\mathrm{CS}}$ for $\mathrm{HCN}$ (4-3) and CS (7-6), respectively. The dense clumps may be under gravitational collapse if $\sigma_{\mathrm{vir}}$ is larger than $\sigma_{\text {line. }}$ The median ratio of $\sigma_{\mathrm{HCN}}$ to $\sigma_{\mathrm{vir}}$ is $\sim 1.2$, and the median ratio of $\sigma_{\mathrm{CS}}$ to $\sigma_{\mathrm{vir}}$ is $\sim 0.8$, indicating that most sources should be in virial equilibrium. There are 45 dense clumps having both $\sigma_{\mathrm{HCN}}$ and $\sigma_{\mathrm{CS}}$ smaller than $\sigma_{\text {vir }}$, indicating that the gas motions may not provide sufficient support against the local gravitational collapse in these clumps. In contrast, there are 37 dense clumps that have both $\sigma_{\mathrm{HCN}}$ and $\sigma_{\mathrm{CS}}$ larger than $\sigma_{\mathrm{vir}}$, indicating that these clumps may be more turbulent and unbound. However, a broad line profile can also be caused by infall. Among the 37 sources, 11 show "blue profile" with blueshifted emission peak stronger than redshifted one, a signature for collapse (Zhou et al. 1993), indicating that these 11 sources may be also bound and probably be in global collapse. Further mapping studies will tell whether they are really in collapse or not.

\subsection{The $\mathrm{K}-\mathrm{S}$ Law Revealed by $1.2 \mathrm{~mm}$ Continuиm}

In panel (a) of Figure 3, we first examine the correlation between total infrared luminosities $\left(L_{\mathrm{TIR}}\right)$ and Clump masses $\left(M_{\text {clump }}\right)$ for Galactic clumps. $L_{\mathrm{TIR}}$ was obtained from fit of the SED (consisting of the $1.2 \mathrm{~mm}$ flux and the fluxes in the four IRAS bands), and $M_{\text {clump }}$ was derived from $1.2 \mathrm{~mm}$ continuum (Faúndez et al. 2004). In Section 4.1, we will show that the $L_{\text {TIR }}$ obtained from integrating the SED in our work are consistent with the total infrared luminosities derived only from the four IRAS bands as used in other works $(\mathrm{Wu}$ et al. 2005, 2010). We use $L_{\mathrm{IR}}$ for the total infrared luminosities derived only from the four IRAS bands to distinguish from the total infrared luminosities derived from SED fit $\left(L_{\mathrm{TIR}}\right)$. The least squares fit to all the data gave a super-linear slope due to the contamination of several low luminosity sources which are located obviously below the fit. The clumps with luminosities lower than $10^{3} L_{\odot}$ are likely low-mass star forming regions. If we only consider the clumps with $L_{\mathrm{TIR}}$ larger than $10^{3} L_{\odot}$, the least squares fit indicates that the relation between $L_{\mathrm{TIR}}$ and $M$ is linear. The linear relation between $L_{\mathrm{TIR}}$ and $M_{\text {clump }}$ can be expressed as:

$$
\frac{L_{\mathrm{TIR}}}{L_{\odot}}=10^{1.80 \pm 0.15} \frac{M_{\text {clump }}}{M_{\odot}} .
$$

Following Kennicutt \& Evans (2012), we can convert $L_{\mathrm{TIR}}$ to a SFR $\left(\dot{M}_{*}\right)$ as:

$$
\log \left(\frac{\dot{M}_{*}}{M_{\odot} \mathrm{yr}^{-1}}\right)=\log \left(\frac{L_{\mathrm{TIR}}}{\operatorname{erg~s}^{-1}}\right)-43.41 .
$$

The use of extragalactic relation between $L_{\mathrm{TIR}}$ and SFR may significantly underestimate the SFR for low-mass molecular clumps, where the initial mass function (IMF) is not fully sampled (Wu et al. 2005; Vutisalchavakul \& Evans 2013). While $L_{\mathrm{TIR}}$ can well trace SFR for dense clumps having $L_{\mathrm{TIR}}$ exceed $10^{4.5} L_{\odot}$ (Wu et al. 2005; Vutisalchavakul $\&$ Evans 2013). In our sample, $71 \%$ sources have $L_{\mathrm{TIR}}$ exceed $10^{4.5} L_{\odot}$ and $92 \%$ have $L_{\mathrm{TIR}}$ exceed $10^{4} L_{\odot}$. In addition, we do not see clear breakup in the $L_{\mathrm{TIR}}$ and $M_{\text {clump }}$ correlation. Therefore, the applying of extragalactic relation on our sample may not severely underestimate the SFR. 

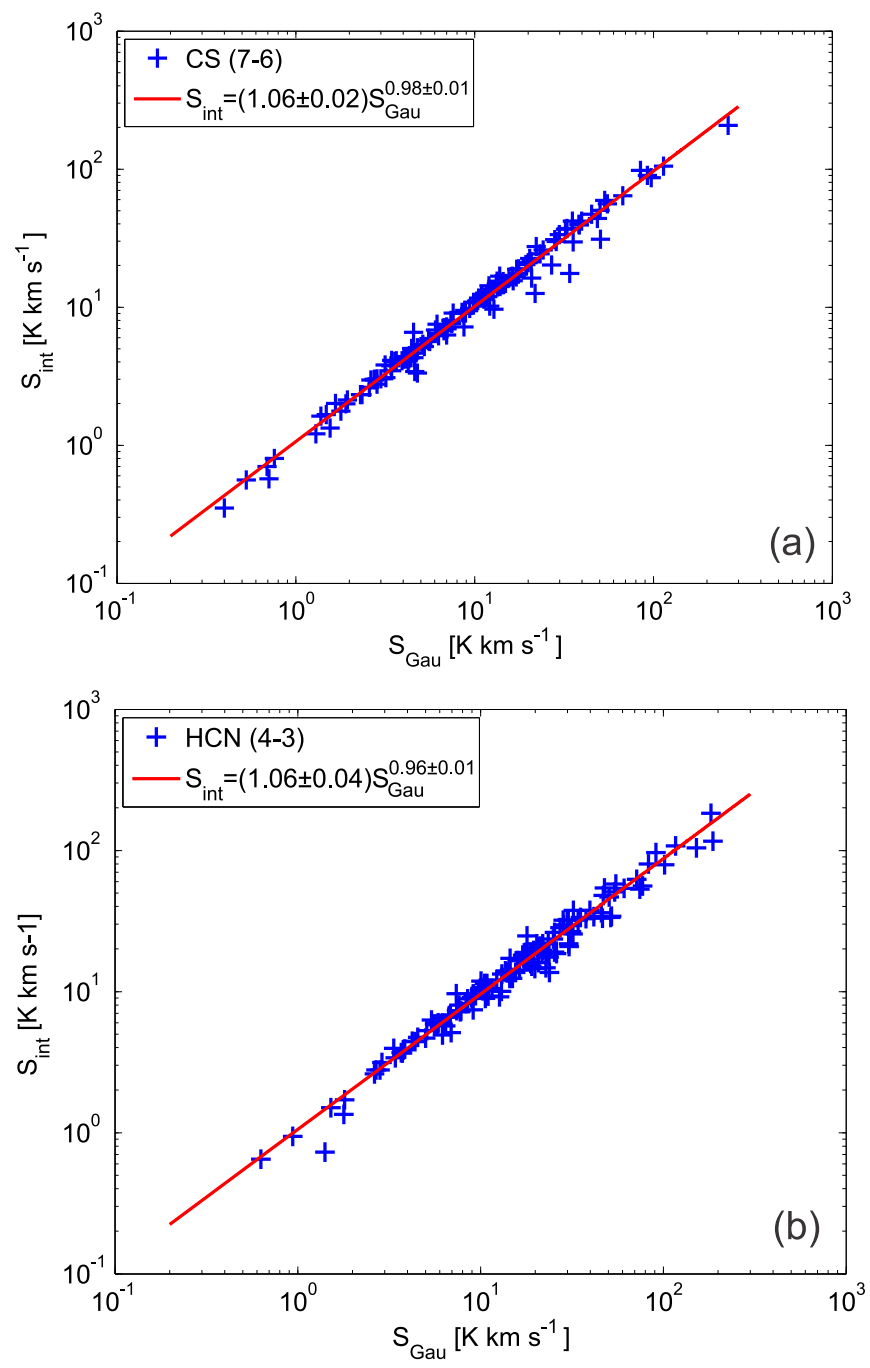

Figure 2. Comparison between the integrated intensities $\left(S_{\mathrm{Gau}}\right)$ from Gaussian fits and the values $\left(S_{\text {int }}\right)$ simply from integrating the lines (Moment 0$)$. The red lines represent the best power-law fits to the data. Panel (a): CS (7-6). Panel (b): HCN (4-3).

From Equations (4) and (5) we can derive the relation between $\dot{M}_{*}$ and $M$ :

$$
\frac{\dot{M}_{*}}{M_{\odot} \mathrm{yr}^{-1}}=\left(0.93_{-0.27}^{+0.38}\right) \times 10^{-8} \frac{M_{\text {clump }}}{M_{\odot}} .
$$

Then, the gas depletion time $\tau_{\text {dep }}$ is:

$$
\tau_{\text {dep }}=\frac{M_{\text {clump }}}{\dot{M}_{*}}=107_{-31}^{+44} \mathrm{Myr} .
$$

Wu et al. (2005) derived a correlation between $\dot{M}_{*}$ and $M_{\text {clump }}$ as $\frac{\dot{M}_{*}}{M_{\odot} \mathrm{yr}^{-1}} \sim 1.2 \times 10^{-8} \frac{M_{\text {clump }}}{M_{\odot}}$, corresponding to a gas depletion time of $\sim 83 \mathrm{Myr}$, which is smaller than the value we derived here. The difference is because that they used a larger conversion factor $\left(\frac{\dot{M}_{*}}{M_{\odot} \mathrm{yr}^{-1}}=2.0 \times 10^{-10} \frac{L_{\mathrm{IR}}}{L_{\odot}}\right)$ between infrared luminosities and SFRs. If we use the same conversion factor, the depletion time should be $\sim 79_{-23}^{+33} \mathrm{Myr}$, which is consistent with their value.

To test the $\mathrm{K}-\mathrm{S}$ law in terms of the corresponding surface densities of SFR $\left(\Sigma_{\text {SFR }}\right)$ and dense gas $\left(\Sigma_{\text {dense }}\right)$, we normalized SFR and $M_{\text {clump }}$ using the sizes of the $1.2 \mathrm{~mm}$ continuum emission from Faúndez et al. (2004) to obtain $\Sigma_{\text {SFR }}$ and $\Sigma_{\text {dense }}$. In panel (b) of Figure 3, we present the derived $\Sigma_{\text {SFR }}$ and $\Sigma_{\text {dense }}$ for the whole sample. The correlation between $\Sigma_{\text {SFR }}$ and $\Sigma_{\text {dense }}$ for the whole sample is nearly linear. But the relation for the sources with $L_{\mathrm{TIR}}$ larger than $10^{3} L_{\odot}$ seems to have sub-linear slope. In general, the $\Sigma_{\mathrm{SFR}}-\Sigma_{\text {dense }}$ correlation is not as tight as the $L_{\mathrm{TIR}}-M_{\text {clump }}$ correlation.

In Figure 4, we investigate the integrated Kennicutt-Schmidt laws (SFR versus gas mass correlations) for both Galactic massive clumps and external galaxies. The external galaxies samples are SFGs and active galactic nuclei (AGNs) with intermediate redshift $(z \sim 0.15)$ from Magdis et al. (2013), Local universe Galaxies (Dunne et al. 2000), high redshift $(z>1)$ SFGs (Scoville et al. 2016) and local ultraluminous infrared galaxies (ULIRGs) from Clements et al. (2010). We convert the total infrared luminosities of these external galaxies to SFR with Equation (5). Their dust masses, which were derived from (sub)millimeter continuum, were converted to gas masses by assuming a constant dust-to-gas ratio of 0.01 (Santini et al. 2014). The dependence of the gas metallicity with dust-to-gas ratio was not considered. This, however, may introduce only a minor effect on correlations because the gas metallicity changes less than a factor of $2-3$, while the dust mass spans 2-3 orders of magnitude for these external galaxies (Santini et al. 2014). As shown by the black line, we extend the SFR versus gas mass correlation of Galactic massive clumps to external galaxies. Interestingly, we find that all external galaxies except for high redshift $(z>1)$ SFGs significantly deviate from the correlation. The intermediate redshift SFGs and AGNs (Magdis et al. 2013), and Local universe Galaxies (Dunne et al. 2000) are located below the correlation, indicating that they have smaller star forming efficiencies or longer gas depletion time than Galactic massive clumps. While ULIRGs, which have very high star forming efficiencies, are located above the correlation. Interestingly, high redshift $(z>1)$ SFGs seem to follow the correlation very well. We find a very tight linear correlation between SFR and gas mass for both Galactic massive clumps and the stacked high redshift SFGs. The linear relation can be expressed as:

$$
\begin{aligned}
& \log \left(\frac{\mathrm{SFR}}{M_{\odot} \mathrm{yr}^{-1}}\right)=(0.97 \pm 0.04) \log \left(\frac{M}{M_{\odot}}\right) \\
& -(7.98 \pm 0.04) ; R=0.99 .
\end{aligned}
$$

This tight relation may indicate a constant molecular gas depletion time of $~ 100$ Myr for both Galactic massive clumps and high redshift SFGs.

\subsection{The Dense Molecular Gas K-S Law}

In panels (a) and (b) of Figure 5, we show the $L_{\mathrm{TIR}}-L_{\mathrm{CS}}^{\prime}$ and $L_{\mathrm{TIR}}-L_{\mathrm{HCN}}^{\prime}$ correlations for Galactic clumps, respectively. Both $L_{\mathrm{TIR}}-L_{\mathrm{CS}}^{\prime}$ and $L_{\mathrm{TIR}}-L_{\mathrm{HCN}}^{\prime}$ correlations are very tight over about four orders of magnitude in $L_{\mathrm{TIR}}$. The best linear leastsquares fits with uncertainties are listed below, with $R$ indicating the correlation coefficient:

$$
\begin{aligned}
\log \left(L_{\mathrm{TIR}}\right)= & (0.84 \pm 0.04) \times \log \left(L^{\prime}{ }_{\mathrm{CS}}\right) \\
& +(3.99 \pm 0.05) ; R=0.88 \\
\log \left(L_{\mathrm{TIR}}\right)= & (0.91 \pm 0.04) \times \log \left(L^{\prime}{ }_{\mathrm{HCN}}\right) \\
& +(3.74 \pm 0.06) ; R=0.87
\end{aligned}
$$


Table 2

Derived Parameters

\begin{tabular}{|c|c|c|c|c|c|c|c|c|}
\hline IRAS Name & $\begin{array}{l}L_{\mathrm{IR}} \\
\left(L_{\odot}\right)\end{array}$ & $\begin{array}{l}L_{\mathrm{TIR}} \\
\left(L_{\odot}\right)\end{array}$ & $\begin{array}{c}L^{\prime}(\mathrm{HCN}) \\
\left(\mathrm{Km} \mathrm{s}^{-1} \mathrm{pc}^{2}\right)\end{array}$ & $\begin{array}{c}L^{\prime}(\mathrm{CS}) \\
\left(\mathrm{K} \mathrm{km} \mathrm{s}^{-1} \mathrm{pc}^{2}\right)\end{array}$ & $\begin{array}{c}\sigma_{\mathrm{vir}} \\
\left(\mathrm{km} \mathrm{s}^{-1}\right)\end{array}$ & $\begin{array}{l}\log (\mathrm{SFR}) \\
\left(M_{\odot} \mathrm{yr}^{-1}\right)\end{array}$ & $\begin{array}{l}\log \left(\sum_{\text {dense }}\right) \\
\left(M_{\odot} \mathrm{pc}^{-2}\right)\end{array}$ & $\begin{array}{c}\log \left(\Sigma_{\mathrm{SFR}}\right) \\
\left(M_{\odot} \mathrm{Myr}^{-1} \mathrm{pc}^{-2}\right)\end{array}$ \\
\hline 08076-3556 & $1.09 \mathrm{E}+01$ & $1.60 \mathrm{E}+01$ & $\ldots$ & $\ldots$ & 0.47 & -8.63 & 3.14 & -0.23 \\
\hline 08303-4303 & $6.79 \mathrm{E}+03$ & $6.70 \mathrm{E}+03$ & $2.84(0.09)$ & $1.75(0.05)$ & 1.52 & -6.00 & 3.50 & 1.08 \\
\hline $08448-4343$ & $6.63 \mathrm{E}+02$ & $1.10 \mathrm{E}+03$ & $0.73(0.01)$ & $0.33(0.01)$ & 0.87 & -6.79 & 3.35 & 0.97 \\
\hline 08470-4243 & $1.23 \mathrm{E}+04$ & $1.10 \mathrm{E}+04$ & $2.59(0.10)$ & $1.35(0.05)$ & 1.45 & -5.79 & 3.47 & 1.32 \\
\hline 09002-4732 & $4.61 \mathrm{E}+04$ & $3.90 \mathrm{E}+04$ & $3.03(0.03)$ & $2.01(0.02)$ & 1.77 & -5.24 & 3.76 & 2.11 \\
\hline $09018-4816$ & $5.05 \mathrm{E}+04$ & $5.20 \mathrm{E}+04$ & $10.64(0.55)$ & $7.63(0.09)$ & 2.52 & -5.11 & 3.81 & 1.70 \\
\hline 09094-4803 & $4.73 \mathrm{E}+04$ & $4.00 \mathrm{E}+04$ & $\ldots$ & $\ldots$ & 1.70 & -5.23 & 2.96 & 0.59 \\
\hline $10365-5803$ & $2.04 \mathrm{E}+04$ & $1.90 \mathrm{E}+04$ & $3.86(0.15)$ & $1.70(0.19)$ & 1.75 & -5.55 & 3.49 & 1.26 \\
\hline $11298-6155$ & $1.91 \mathrm{E}+05$ & $1.70 \mathrm{E}+05$ & $24.65(0.96)$ & $16.04(0.90)$ & 2.39 & -4.60 & 3.27 & 1.24 \\
\hline $11332-6258$ & $5.42 \mathrm{E}+03$ & $5.30 \mathrm{E}+03$ & $0.31(0.05)$ & $0.29(0.03)$ & 1.16 & -6.11 & 3.34 & 1.12 \\
\hline $11590-6452$ & $3.85 \mathrm{E}+01$ & $5.30 \mathrm{E}+01$ & $0.01(0.00)$ & $\ldots$ & 0.80 & -8.11 & 3.69 & 0.47 \\
\hline $12320-6122$ & $2.64 \mathrm{E}+05$ & $2.20 \mathrm{E}+05$ & $25.87(0.69)$ & $16.08(0.32)$ & 2.84 & -4.49 & 3.68 & 1.87 \\
\hline $12326-6245$ & $3.31 \mathrm{E}+05$ & $2.70 \mathrm{E}+05$ & $68.27(0.70)$ & $56.13(0.30)$ & 3.23 & -4.40 & 3.84 & 2.07 \\
\hline $12383-6128$ & $4.95 \mathrm{E}+04$ & $4.90 \mathrm{E}+04$ & $19.43(0.55)$ & $14.49(0.48)$ & 1.90 & -5.14 & 3.10 & 0.76 \\
\hline $12572-6316$ & $5.43 \mathrm{E}+03$ & $5.60 \mathrm{E}+03$ & $2.18(0.09)$ & $1.22(0.07)$ & 1.25 & -6.08 & 3.15 & 0.64 \\
\hline $13079-6218$ & $2.42 \mathrm{E}+05$ & $2.80 \mathrm{E}+05$ & $85.68(1.07)$ & $54.36(0.58)$ & 3.52 & -4.38 & 3.82 & 1.88 \\
\hline $13080-6229$ & $1.95 \mathrm{E}+05$ & $3.20 \mathrm{E}+05$ & $14.96(0.27)$ & $7.73(0.15)$ & 1.92 & -4.32 & 3.45 & 2.26 \\
\hline $13111-6228$ & $1.47 \mathrm{E}+05$ & $1.30 \mathrm{E}+05$ & $28.59(0.38)$ & $23.08(0.22)$ & 2.37 & -4.72 & 3.49 & 1.57 \\
\hline $13134-6242$ & $3.51 \mathrm{E}+04$ & $3.10 \mathrm{E}+04$ & $11.01(0.36)$ & $5.69(0.09)$ & 1.97 & -5.34 & 3.66 & 1.61 \\
\hline $13140-6226$ & $7.99 \mathrm{E}+03$ & $7.80 \mathrm{E}+03$ & $5.90(0.16)$ & $2.71(0.13)$ & 1.40 & -5.94 & 3.29 & 0.86 \\
\hline $13291-6229$ & $5.23 \mathrm{E}+04$ & $4.40 \mathrm{E}+04$ & $7.35(0.35)$ & $4.77(0.10)$ & 1.20 & -5.19 & 2.96 & 1.23 \\
\hline $13291-6249$ & $2.81 \mathrm{E}+04$ & $2.50 \mathrm{E}+04$ & $9.41(0.06)$ & $5.46(0.06)$ & 1.62 & -5.43 & 3.47 & 1.48 \\
\hline $13295-6152$ & $6.50 \mathrm{E}+03$ & $7.00 \mathrm{E}+03$ & $3.23(0.29)$ & $\ldots$ & 1.56 & -5.98 & 3.10 & 0.26 \\
\hline $13471-6120$ & $3.12 \mathrm{E}+05$ & $2.70 \mathrm{E}+05$ & $23.50(0.30)$ & $16.80(0.21)$ & 2.70 & -4.40 & 3.62 & 1.92 \\
\hline $13484-6100$ & $8.25 \mathrm{E}+04$ & $7.10 \mathrm{E}+04$ & $34.69(1.04)$ & $23.53(0.49)$ & 2.06 & -4.98 & 3.40 & 1.38 \\
\hline $14013-6105$ & $2.04 \mathrm{E}+05$ & $1.70 \mathrm{E}+05$ & $61.46(1.41)$ & $35.19(0.26)$ & 2.54 & -4.60 & 3.53 & 1.66 \\
\hline $14050-6056$ & $\ldots$ & $7.20 \mathrm{E}+04$ & $9.61(0.18)$ & $7.76(0.12)$ & 1.83 & -4.97 & 3.35 & 1.49 \\
\hline $14164-6028$ & $1.02 \mathrm{E}+04$ & $9.30 \mathrm{E}+03$ & $4.50(0.14)$ & $2.74(0.06)$ & 0.90 & -5.86 & 2.90 & 0.92 \\
\hline $14206-6151$ & $1.29 \mathrm{E}+04$ & $1.30 \mathrm{E}+04$ & $1.08(0.13)$ & $0.46(0.13)$ & 1.46 & -5.72 & 3.15 & 0.75 \\
\hline $14212-6131$ & $1.74 \mathrm{E}+04$ & $1.20 \mathrm{E}+05$ & $13.25(0.39)$ & $4.47(0.29)$ & 2.28 & -4.75 & 3.51 & 1.64 \\
\hline $14382-6017$ & $8.55 \mathrm{E}+04$ & $7.50 \mathrm{E}+04$ & $15.68(0.81)$ & $10.59(0.26)$ & 1.62 & -4.95 & 3.11 & 1.23 \\
\hline $14453-5912$ & $2.98 \mathrm{E}+04$ & $2.80 \mathrm{E}+04$ & $7.16(0.33)$ & $3.88(0.08)$ & 1.51 & -5.38 & 3.28 & 1.27 \\
\hline $14498-5856$ & $3.87 \mathrm{E}+04$ & $3.50 \mathrm{E}+04$ & $18.71(0.31)$ & $11.41(0.17)$ & 2.11 & -5.29 & 3.55 & 1.32 \\
\hline $15122-5801$ & $8.09 \mathrm{E}+04$ & $7.20 \mathrm{E}+04$ & $5.02(0.19)$ & $3.78(0.17)$ & 1.71 & -4.97 & 3.26 & 1.42 \\
\hline $15254-5621$ & $1.70 \mathrm{E}+05$ & $1.20 \mathrm{E}+05$ & $24.66(0.28)$ & $14.49(0.21)$ & 2.74 & -4.75 & 3.66 & 1.63 \\
\hline $15290-5546$ & $4.56 \mathrm{E}+05$ & $3.90 \mathrm{E}+05$ & $78.59(1.76)$ & $55.54(0.58)$ & 3.32 & -4.24 & 3.71 & 1.90 \\
\hline $15384-5348$ & $1.22 \mathrm{E}+05$ & $1.00 \mathrm{E}+05$ & $8.19(0.20)$ & $4.81(0.13)$ & 2.06 & -4.83 & 3.40 & 1.53 \\
\hline $15394-5358$ & $1.50 \mathrm{E}+04$ & $1.50 \mathrm{E}+04$ & $20.68(0.45)$ & $7.73(0.10)$ & 3.39 & -5.65 & 4.06 & 1.15 \\
\hline $15408-5356$ & $2.24 \mathrm{E}+05$ & $1.90 \mathrm{E}+05$ & $43.85(0.81)$ & $28.67(0.98)$ & 2.76 & -4.55 & 3.75 & 1.99 \\
\hline $15411-5352$ & $1.57 \mathrm{E}+05$ & $1.40 \mathrm{E}+05$ & $13.39(0.24)$ & $6.37(0.11)$ & 2.19 & -4.68 & 3.66 & 2.09 \\
\hline $15437-5343$ & $6.72 \mathrm{E}+04$ & $6.10 \mathrm{E}+04$ & $2.64(0.26)$ & $\ldots$ & 1.70 & -5.04 & 3.22 & 1.27 \\
\hline $15439-5449$ & $4.35 \mathrm{E}+04$ & $4.40 \mathrm{E}+04$ & $7.48(0.28)$ & $3.75(0.07)$ & 1.73 & -5.19 & 3.39 & 1.45 \\
\hline $15502-5302$ & $8.90 \mathrm{E}+05$ & $7.50 \mathrm{E}+05$ & $46.91(0.48)$ & $31.70(0.37)$ & 3.52 & -3.95 & 3.84 & 2.36 \\
\hline $15520-5234$ & $2.23 \mathrm{E}+05$ & $2.00 \mathrm{E}+05$ & $52.64(0.32)$ & $47.85(0.13)$ & 2.73 & -4.53 & 3.90 & 2.33 \\
\hline $15522-5411$ & $2.22 \mathrm{E}+04$ & $2.10 \mathrm{E}+04$ & $6.46(0.60)$ & $5.66(0.27)$ & 1.38 & -5.51 & 3.05 & 0.85 \\
\hline $15557-5215$ & $2.00 \mathrm{E}+04$ & $2.30 \mathrm{E}+04$ & $12.81(0.43)$ & $5.30(0.30)$ & 2.69 & -5.47 & 3.68 & 0.98 \\
\hline $15567-5236$ & $9.79 \mathrm{E}+05$ & $8.00 \mathrm{E}+05$ & $85.65(0.93)$ & $69.97(0.66)$ & 4.50 & -3.93 & 3.95 & 2.16 \\
\hline $15570-5227$ & $1.35 \mathrm{E}+05$ & $1.60 \mathrm{E}+05$ & $14.73(0.41)$ & $11.85(0.26)$ & 2.20 & -4.63 & 3.31 & 1.42 \\
\hline $15584-5247$ & $3.93 \mathrm{E}+04$ & $3.60 \mathrm{E}+04$ & $6.02(0.41)$ & $3.38(0.37)$ & 1.79 & -5.27 & 3.16 & 0.85 \\
\hline $15596-5301$ & $6.71 \mathrm{E}+04$ & $6.40 \mathrm{E}+04$ & $33.72(2.56)$ & $22.98(0.38)$ & 2.25 & -5.02 & 3.49 & 1.35 \\
\hline $16026-5035$ & $1.64 \mathrm{E}+05$ & $1.40 \mathrm{E}+05$ & $13.37(0.79)$ & $8.67(0.35)$ & 1.77 & -4.68 & 3.07 & 1.27 \\
\hline $16037-5223$ & $1.31 \mathrm{E}+05$ & $1.10 \mathrm{E}+05$ & $20.44(0.45)$ & $19.67(0.34)$ & 2.81 & -4.79 & 3.71 & 1.64 \\
\hline $16060-5146$ & $7.48 \mathrm{E}+05$ & $7.50 \mathrm{E}+05$ & $48.01(2.20)$ & $27.09(0.84)$ & 5.21 & -3.95 & 4.22 & 2.44 \\
\hline $16065-5158$ & $3.03 \mathrm{E}+05$ & $2.90 \mathrm{E}+05$ & $48.91(1.06)$ & $45.44(0.32)$ & 3.78 & -4.37 & 4.07 & 2.27 \\
\hline $16071-5142$ & $7.80 \mathrm{E}+04$ & $8.30 \mathrm{E}+04$ & $79.40(2.81)$ & $49.59(0.55)$ & 3.68 & -4.91 & 3.89 & 1.41 \\
\hline $16076-5134$ & $2.26 \mathrm{E}+05$ & $2.00 \mathrm{E}+05$ & $116.84(1.13)$ & $91.94(1.25)$ & 2.79 & -4.53 & 3.56 & 1.61 \\
\hline $16119-5048$ & $3.47 \mathrm{E}+04$ & $3.30 \mathrm{E}+04$ & $15.82(0.50)$ & $8.86(0.18)$ & 2.14 & -5.31 & 3.60 & 1.37 \\
\hline $16132-5039$ & $7.20 \mathrm{E}+04$ & $6.30 \mathrm{E}+04$ & $3.91(0.15)$ & $2.07(0.10)$ & 1.65 & -5.03 & 3.26 & 1.43 \\
\hline $16158-5055$ & $1.99 \mathrm{E}+05$ & $1.60 \mathrm{E}+05$ & $15.88(0.39)$ & $11.39(0.24)$ & 2.56 & -4.63 & 3.52 & 1.60 \\
\hline $16164-5046$ & $4.51 \mathrm{E}+05$ & $4.00 \mathrm{E}+05$ & $56.74(1.18)$ & $49.60(0.30)$ & 4.35 & -4.23 & 4.24 & 2.51 \\
\hline $16172-5028$ & $4.69 \mathrm{E}+05$ & $4.40 \mathrm{E}+05$ & $87.43(1.14)$ & $112.92(1.17)$ & 4.23 & -4.19 & 3.82 & 1.77 \\
\hline $16177-5018$ & $3.83 \mathrm{E}+05$ & $3.40 \mathrm{E}+05$ & $4.79(0.44)$ & $3.71(0.32)$ & 4.66 & -4.30 & 4.26 & 2.36 \\
\hline $16272-4837$ & $2.52 \mathrm{E}+04$ & $3.00 \mathrm{E}+04$ & $22.32(0.62)$ & $9.11(0.15)$ & 2.59 & -5.35 & 3.77 & 1.34 \\
\hline
\end{tabular}


Table 2

(Continued)

\begin{tabular}{|c|c|c|c|c|c|c|c|c|}
\hline IRAS Name & $\begin{array}{l}L_{\mathrm{IR}} \\
\left(L_{\odot}\right)\end{array}$ & $\begin{array}{l}L_{\mathrm{TIR}} \\
\left(L_{\odot}\right)\end{array}$ & $\begin{array}{c}L^{\prime}(\mathrm{HCN}) \\
\left(\mathrm{K} \mathrm{km} \mathrm{s}^{-1} \mathrm{pc}^{2}\right)\end{array}$ & $\begin{array}{c}L^{\prime}(\mathrm{CS}) \\
\left(\mathrm{K} \mathrm{km} \mathrm{s}^{-1} \mathrm{pc}^{2}\right)\end{array}$ & $\begin{array}{c}\sigma_{\mathrm{vir}} \\
\left(\mathrm{km} \mathrm{s}^{-1}\right)\end{array}$ & $\begin{array}{l}\log (\mathrm{SFR}) \\
\left(M_{\odot} \mathrm{yr}^{-1}\right)\end{array}$ & $\begin{array}{l}\log \left(\sum_{\text {dense }}\right) \\
\left(M_{\odot} \mathrm{pc}^{-2}\right)\end{array}$ & $\begin{array}{c}\log \left(\Sigma_{\mathrm{SFR}}\right) \\
\left(M_{\odot} \mathrm{Myr}^{-1} \mathrm{pc}^{-2}\right)\end{array}$ \\
\hline $16297-4757$ & $5.77 \mathrm{E}+05$ & $5.10 \mathrm{E}+05$ & $87.74(2.00)$ & $105.74(1.33)$ & 3.28 & -4.12 & 3.43 & 1.49 \\
\hline $16304-4710$ & $1.08 \mathrm{E}+05$ & $1.00 \mathrm{E}+05$ & $16.42(1.09)$ & $6.89(1.00)$ & 1.86 & -4.83 & 2.93 & 0.75 \\
\hline $16313-4729$ & $1.09 \mathrm{E}+06$ & $9.30 \mathrm{E}+05$ & $73.41(2.00)$ & $37.58(2.00)$ & 3.41 & -3.86 & 3.35 & 1.52 \\
\hline $16318-4724$ & $2.64 \mathrm{E}+05$ & $2.50 \mathrm{E}+05$ & $38.09(0.96)$ & $28.61(0.42)$ & 3.20 & -4.43 & 3.71 & 1.77 \\
\hline $16330-4725$ & $2.27 \mathrm{E}+06$ & $1.90 \mathrm{E}+06$ & $297.54(2.59)$ & $222.09(1.98)$ & 3.01 & -3.55 & 3.19 & 1.73 \\
\hline $16344-4658$ & $5.28 \mathrm{E}+04$ & $5.10 \mathrm{E}+04$ & $21.15(0.50)$ & $16.60(0.70)$ & 2.26 & -5.12 & 3.49 & 1.25 \\
\hline $16348-4654$ & $5.45 \mathrm{E}+05$ & $6.50 \mathrm{E}+05$ & $264.00(4.19)$ & $147.83(1.76)$ & 5.58 & -4.02 & 3.92 & 1.65 \\
\hline $16351-4722$ & $1.09 \mathrm{E}+05$ & $9.30 \mathrm{E}+04$ & $36.73(0.69)$ & $33.25(0.40)$ & 2.34 & -4.86 & 3.73 & 1.94 \\
\hline $16362-4639$ & $2.01 \mathrm{E}+04$ & $1.70 \mathrm{E}+04$ & $3.19(0.15)$ & $0.63(0.08)$ & 0.96 & -5.60 & 2.75 & 0.79 \\
\hline $16372-4545$ & $3.46 \mathrm{E}+04$ & $3.70 \mathrm{E}+04$ & $15.91(0.49)$ & $13.04(0.39)$ & 1.30 & -5.26 & 2.99 & 1.06 \\
\hline $16385-4619$ & $1.46 \mathrm{E}+05$ & $1.30 \mathrm{E}+05$ & $51.47(0.72)$ & $46.73(0.39)$ & 2.33 & -4.72 & 3.43 & 1.48 \\
\hline $16424-4531$ & $1.92 \mathrm{E}+04$ & $1.90 \mathrm{E}+04$ & $13.10(0.23)$ & $2.70(0.14)$ & 1.82 & -5.55 & 3.42 & 1.06 \\
\hline $16445-4459$ & $1.18 \mathrm{E}+05$ & $1.10 \mathrm{E}+05$ & $26.91(0.60)$ & $11.81(0.39)$ & 2.34 & -4.79 & 3.41 & 1.37 \\
\hline $16458-4512$ & $4.99 \mathrm{E}+04$ & $5.10 \mathrm{E}+04$ & $36.72(0.76)$ & $9.64(0.51)$ & 2.54 & -5.12 & 3.47 & 1.00 \\
\hline $16484-4603$ & $6.27 \mathrm{E}+04$ & $5.50 \mathrm{E}+04$ & $16.89(0.42)$ & $10.99(0.16)$ & 1.90 & -5.09 & 3.49 & 1.58 \\
\hline $16487-4423$ & $4.79 \mathrm{E}+04$ & $3.00 \mathrm{E}+04$ & $8.89(0.22)$ & $3.09(0.14)$ & 1.64 & -5.35 & 3.20 & 1.01 \\
\hline $16489-4431$ & $1.72 \mathrm{E}+04$ & $1.50 \mathrm{E}+04$ & $5.44(0.16)$ & $2.41(0.10)$ & 1.53 & -5.65 & 3.25 & 0.93 \\
\hline $16506-4512$ & $1.17 \mathrm{E}+05$ & $1.10 \mathrm{E}+05$ & $22.55(0.44)$ & $13.86(0.24)$ & 2.34 & -4.79 & 3.52 & 1.59 \\
\hline $16524-4300$ & $4.50 \mathrm{E}+04$ & $4.20 \mathrm{E}+04$ & $17.29(0.34)$ & $8.93(0.10)$ & 2.54 & -5.21 & 3.70 & 1.38 \\
\hline $16547-4247$ & $6.47 \mathrm{E}+04$ & $6.30 \mathrm{E}+04$ & $68.73(0.76)$ & $31.36(0.22)$ & 2.94 & -5.03 & 3.92 & 1.74 \\
\hline $16562-3959$ & $5.63 \mathrm{E}+04$ & $4.80 \mathrm{E}+04$ & $16.66(0.11)$ & $8.94(0.05)$ & 3.71 & -5.15 & 4.29 & 1.97 \\
\hline $16571-4029$ & $8.81 \mathrm{E}+04$ & $7.60 \mathrm{E}+04$ & $17.01(0.92)$ & $11.65(0.22)$ & 1.51 & -4.95 & 3.21 & 1.56 \\
\hline $17006-4215$ & $4.58 \mathrm{E}+04$ & $4.10 \mathrm{E}+04$ & $6.33(0.10)$ & $5.33(0.05)$ & 2.29 & -5.22 & 3.81 & 1.76 \\
\hline $17008-4040$ & $6.47 \mathrm{E}+04$ & $5.20 \mathrm{E}+04$ & $10.96(0.23)$ & $6.85(0.08)$ & 3.42 & -5.11 & 4.15 & 1.86 \\
\hline $17016-4124$ & $6.73 \mathrm{E}+04$ & $5.90 \mathrm{E}+04$ & $36.08(0.62)$ & $14.23(0.18)$ & 3.53 & -5.06 & 4.15 & 1.86 \\
\hline $17136-3617$ & $1.17 \mathrm{E}+05$ & $1.10 \mathrm{E}+05$ & $8.11(0.05)$ & $6.17(0.03)$ & 2.46 & -4.79 & 3.90 & 2.25 \\
\hline $17143-3700$ & $4.72 \mathrm{E}+04$ & $4.10 \mathrm{E}+04$ & $10.43(0.24)$ & $6.94(0.12)$ & 1.37 & -5.22 & 3.17 & 1.39 \\
\hline $17158-3901$ & $2.11 \mathrm{E}+04$ & $2.20 \mathrm{E}+04$ & $9.05(0.17)$ & $5.22(0.04)$ & 2.62 & -5.49 & 4.01 & 1.66 \\
\hline $17160-3707$ & $4.47 \mathrm{E}+05$ & $4.30 \mathrm{E}+05$ & $122.66(2.86)$ & $69.94(0.93)$ & 4.59 & -4.20 & 3.72 & 1.41 \\
\hline $17175-3544$ & $7.56 \mathrm{E}+04$ & $7.80 \mathrm{E}+04$ & $12.06(0.16)$ & $11.23(0.03)$ & 3.32 & -4.94 & 4.37 & 2.52 \\
\hline $17204-3636$ & $1.51 \mathrm{E}+04$ & $1.50 \mathrm{E}+04$ & $10.29(0.58)$ & $4.30(0.11)$ & 1.86 & -5.65 & 3.47 & 1.01 \\
\hline $17220-3609$ & $5.45 \mathrm{E}+05$ & $6.80 \mathrm{E}+05$ & $304.01(2.91)$ & $256.19(1.16)$ & 6.46 & -4.00 & 4.05 & 1.67 \\
\hline $17233-3606$ & $1.59 \mathrm{E}+04$ & $1.70 \mathrm{E}+04$ & 6.57(0.03) & $4.10(0.03)$ & 2.35 & -5.60 & 4.36 & 2.43 \\
\hline $17244-3536$ & $2.11 \mathrm{E}+04$ & $1.90 \mathrm{E}+04$ & $2.55(0.06)$ & $1.25(0.05)$ & 1.37 & -5.55 & 3.28 & 1.29 \\
\hline $17258-3637$ & $2.12 \mathrm{E}+05$ & $1.90 \mathrm{E}+05$ & $17.92(0.58)$ & $13.75(0.11)$ & 3.28 & -4.55 & 4.11 & 2.41 \\
\hline $17269-3312$ & $5.52 \mathrm{E}+04$ & $4.90 \mathrm{E}+04$ & $4.50(0.29)$ & $0.92(0.11)$ & 1.68 & -5.14 & 3.24 & 1.26 \\
\hline $17271-3439$ & $2.78 \mathrm{E}+05$ & $2.60 \mathrm{E}+05$ & $38.82(3.25)$ & $31.15(0.42)$ & 5.32 & -4.42 & 4.24 & 1.98 \\
\hline $17278-3541$ & $6.07 \mathrm{E}+03$ & $5.80 \mathrm{E}+03$ & $2.29(0.07)$ & $0.99(0.04)$ & 1.45 & -6.07 & 3.38 & 0.87 \\
\hline $17439-2845$ & $5.14 \mathrm{E}+05$ & $4.80 \mathrm{E}+05$ & $33.53(1.11)$ & $11.52(0.96)$ & 3.13 & -4.15 & 3.39 & 1.45 \\
\hline $17441-2822$ & $3.70 \mathrm{E}+06$ & $3.70 \mathrm{E}+06$ & $2489.25(536.10)$ & $3501.96(16.40)$ & 16.89 & -3.26 & 4.70 & 2.04 \\
\hline $17455-2800$ & $8.67 \mathrm{E}+05$ & $8.10 \mathrm{E}+05$ & $191.52(1.67)$ & $114.47(1.45)$ & 4.13 & -3.92 & 3.54 & 1.51 \\
\hline $17545-2357$ & $1.55 \mathrm{E}+04$ & $1.40 \mathrm{E}+04$ & $4.52(0.09)$ & $2.00(0.06)$ & 1.54 & -5.68 & 3.26 & 0.89 \\
\hline $17589-2312$ & $2.34 \mathrm{E}+04$ & $2.10 \mathrm{E}+04$ & $5.88(0.42)$ & $1.52(0.06)$ & 1.06 & -5.51 & 2.97 & 1.14 \\
\hline $17599-2148$ & $1.18 \mathrm{E}+05$ & $1.10 \mathrm{E}+05$ & $14.09(0.47)$ & $6.98(0.10)$ & 2.84 & -4.79 & 3.72 & 1.66 \\
\hline $18032-2032$ & $3.72 \mathrm{E}+05$ & $3.20 \mathrm{E}+05$ & $91.19(1.49)$ & $61.56(0.88)$ & 3.28 & -4.32 & 3.77 & 1.97 \\
\hline $18056-1952$ & $3.58 \mathrm{E}+05$ & $3.50 \mathrm{E}+05$ & $113.09(2.45)$ & $59.06(0.94)$ & 3.67 & -4.29 & 3.82 & 1.91 \\
\hline $18075-2040$ & $2.46 \mathrm{E}+04$ & $2.40 \mathrm{E}+04$ & $\ldots$ & $\ldots$ & 1.44 & -5.45 & 2.99 & 0.70 \\
\hline $18079-1756$ & $2.42 \mathrm{E}+04$ & $2.00 \mathrm{E}+04$ & 7.51(0.09) & $4.62(0.05)$ & 0.66 & -5.53 & 2.61 & 1.24 \\
\hline $18089-1732$ & $5.13 \mathrm{E}+04$ & $5.20 \mathrm{E}+04$ & $21.91(0.81)$ & $13.86(0.12)$ & 3.01 & -5.11 & 3.87 & 1.53 \\
\hline $18110-1854$ & $1.09 \mathrm{E}+05$ & $9.60 \mathrm{E}+04$ & $19.42(0.30)$ & $9.17(0.08)$ & 2.30 & -4.85 & 3.60 & 1.71 \\
\hline $18116-1646$ & $2.06 \mathrm{E}+05$ & $1.80 \mathrm{E}+05$ & $35.09(0.78)$ & $32.10(1.79)$ & 2.84 & -4.57 & 3.68 & 1.78 \\
\hline $18117-1753$ & $1.11 \mathrm{E}+05$ & $1.10 \mathrm{E}+05$ & $37.47(0.95)$ & $22.83(0.31)$ & 3.13 & -4.79 & 3.83 & 1.70 \\
\hline 18134-1942 & $6.56 \mathrm{E}+03$ & $6.80 \mathrm{E}+03$ & $3.87(0.04)$ & $1.84(0.03)$ & 1.45 & -6.00 & 3.37 & 0.91 \\
\hline $18139-1842$ & $6.62 \mathrm{E}+04$ & $5.80 \mathrm{E}+04$ & $14.68(0.94)$ & $8.37(0.13)$ & 1.38 & -5.07 & 3.12 & 1.43 \\
\hline $18159-1648$ & $2.18 \mathrm{E}+04$ & $2.50 \mathrm{E}+04$ & $16.51(0.32)$ & $11.67(0.05)$ & 3.14 & -5.43 & 4.06 & 1.52 \\
\hline $18182-1433$ & $2.69 \mathrm{E}+04$ & $2.80 \mathrm{E}+04$ & $16.30(0.52)$ & $7.62(0.27)$ & 1.90 & -5.38 & 3.43 & 1.18 \\
\hline $18223-1243$ & $2.50 \mathrm{E}+04$ & $2.70 \mathrm{E}+04$ & $5.06(0.18)$ & $2.20(0.12)$ & 1.95 & -5.40 & 3.36 & 0.97 \\
\hline $18228-1312$ & $5.84 \mathrm{E}+04$ & $5.90 \mathrm{E}+04$ & $9.16(0.35)$ & $3.85(0.27)$ & 1.91 & -5.06 & 3.44 & 1.52 \\
\hline $18236-1205$ & $6.92 \mathrm{E}+03$ & $8.20 \mathrm{E}+03$ & $10.81(0.29)$ & $3.85(0.16)$ & 2.10 & -5.92 & 3.58 & 0.78 \\
\hline $18264-1152$ & $1.38 \mathrm{E}+04$ & $1.60 \mathrm{E}+04$ & $23.24(0.42)$ & $8.69(0.15)$ & 2.53 & -5.63 & 3.76 & 1.09 \\
\hline $18290-0924$ & $3.23 \mathrm{E}+04$ & $3.40 \mathrm{E}+04$ & $\ldots$ & $\ldots$ & 1.97 & -5.30 & 3.26 & 0.84 \\
\hline $18308-0503$ & $2.30 \mathrm{E}+04$ & $2.10 \mathrm{E}+04$ & $2.47(0.12)$ & $0.61(0.06)$ & 1.40 & -5.51 & 3.17 & 1.06 \\
\hline $18311-0809$ & $3.90 \mathrm{E}+05$ & $3.60 \mathrm{E}+05$ & $103.25(3.62)$ & $33.59(0.83)$ & 3.30 & -4.27 & 3.44 & 1.35 \\
\hline
\end{tabular}


Table 2

(Continued)

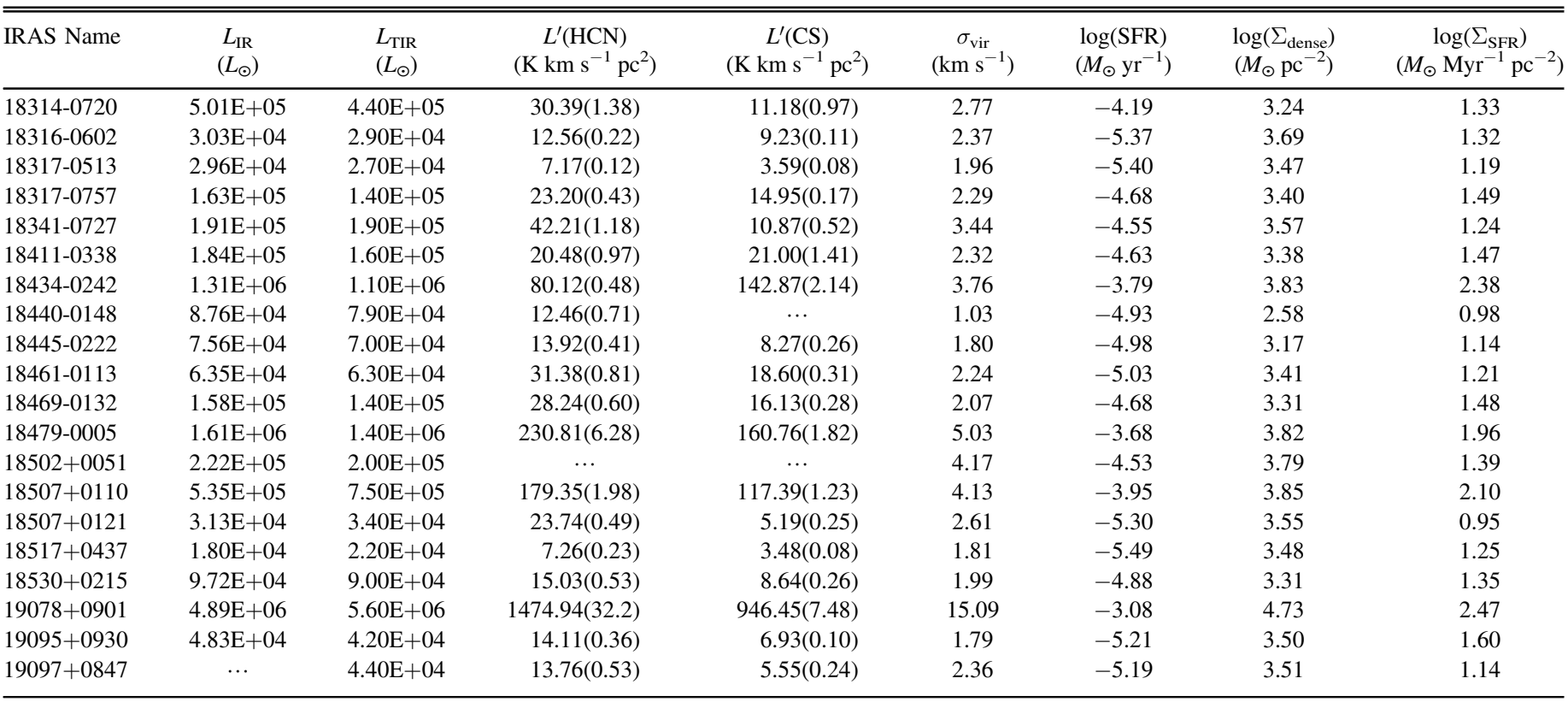
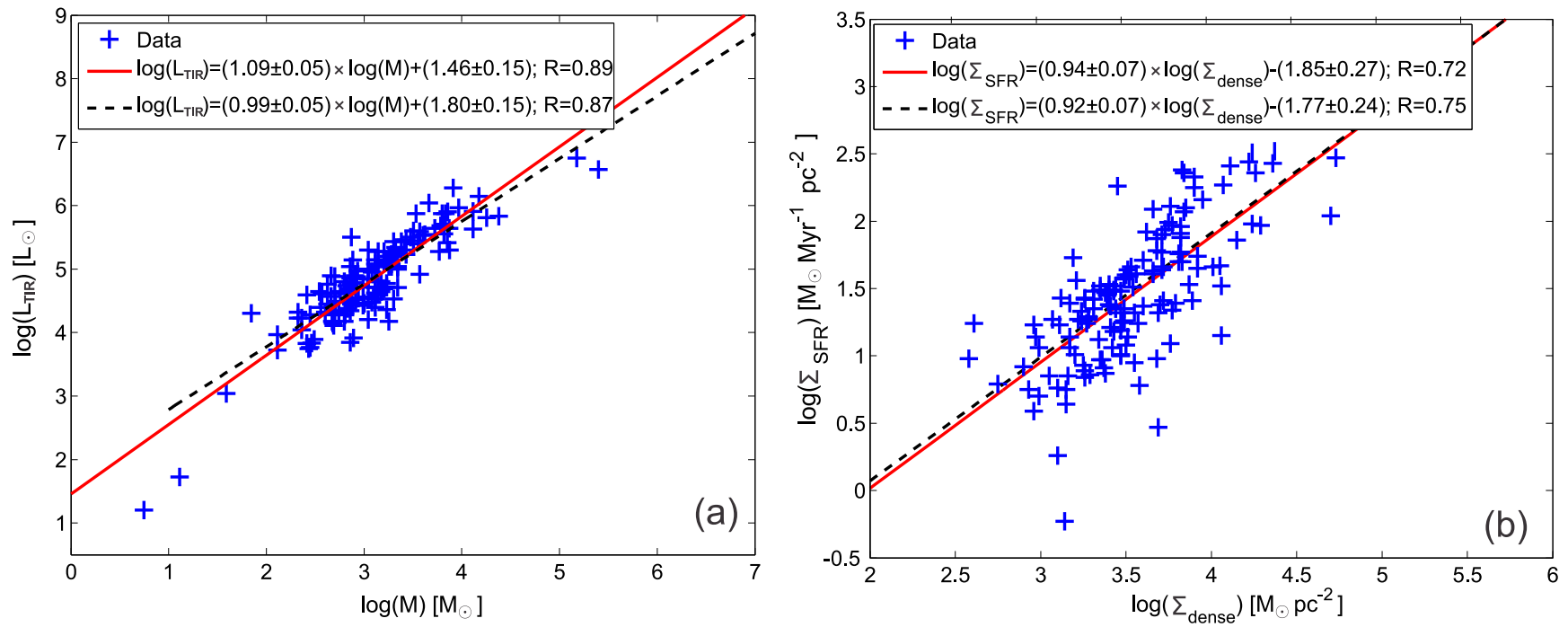

Figure 3. (a). Infrared luminosities $L_{\mathrm{TIR}}$ vs. Clump masses $M$ (for simplicity, hereafter we use $M$ rather than $M_{\text {clump }}$ in the figures). The red line represents least squares fit to all the sources. The black dashed line represents least squares fit to the clumps with $L_{\mathrm{TIR}}$ larger than $10^{3} L_{\odot}$. (b). Surface density of SFR, $\Sigma_{\mathrm{SFR}}$, against surface density of the dense molecular gas mass as traced by the $1.2 \mathrm{~mm}$ continuum, $\Sigma_{\text {dense. }}$. The red line represents least squares fit to all the sources. The black dashed line represents least squares fit to the clumps with $L_{\mathrm{TIR}}$ larger than $10^{3} L_{\odot}$.

From Bayesian regression with LINMIX_ERR (Kelly 2007), the slopes of $L_{\mathrm{TIR}}-L_{\mathrm{CS}}^{\prime}$ and $L_{\mathrm{TIR}}-L_{\mathrm{HCN}}^{\prime}$ are $0.85 \pm 0.04$ and $0.93 \pm 0.05$, respectively, which are consistent with results from linear least-squares fits. The relations are sub-linear. $\mathrm{Wu}$ et al. (2010) found a slope of $0.81 \pm 0.04$ for $L_{\mathrm{IR}}-L_{\mathrm{CS}}^{\prime}$ relation, which is similar to the slope derived for our sample. However, as shown in green dashed line in panel (a), the intercept in their correlation is larger than ours. We will discuss this inconsistence in Section 4.1.

A threshold in luminosity at $L_{\mathrm{IR}}=10^{4.5} L_{\odot}$ was found for the correlations between $L_{\mathrm{IR}}$ and molecular line luminosity from observations of lower $J$ transition lines ( $\mathrm{Wu}$ et al. 2005, 2010). Above the threshold, a nearly linear correlation was found between the infrared luminosity and the line luminosity of all dense gas tracers for Galactic dense clumps (Wu et al. 2005, 2010). In Figure 6, the distance independent ratio $L_{\mathrm{TIR}} / L_{\text {mol }}^{\prime}$ has been plotted versus $L_{\mathrm{TIR}}$. In general, sources with $L_{\mathrm{TIR}}>10^{4.5} L_{\odot}$ have slightly larger $L_{\mathrm{TIR}} / L_{\mathrm{mol}}^{\prime}$ than sources with $L_{\mathrm{TIR}}<10^{4.5} L_{\odot}$. The median log $\left(L_{\mathrm{TIR}} / L_{\mathrm{HCN}}^{\prime}\right)$ values for sources with $L_{\mathrm{TIR}}>10^{4.5} L_{\odot}$ and with $L_{\mathrm{TIR}}<10^{4.5} L_{\odot}$ are $\sim 3.70 \pm 0.25$ and $\sim 3.42 \pm 0.48$, respectively. The uncertainties are the standard deviation of the means. The median $\log \left(L_{\mathrm{TIR}} / L_{\mathrm{CS}}^{\prime}\right)$ values for sources with $L_{\mathrm{TIR}}>10^{4.5} L_{\odot}$ and with $L_{\mathrm{TIR}}<10^{4.5} L_{\odot}$ are $\sim 3.88 \pm 0.14$ and $\sim 3.66 \pm 0.15$, respectively. Therefore, considering uncertainties, there seems no threshold for HCN (4-3) and CS (7-6) lines in $L_{\mathrm{TIR}}-L_{\text {mol }}^{\prime}$ correlations in our sample. The correlations of $L_{\mathrm{TIR}}-L_{\mathrm{CS}}^{\prime}$ and $L_{\mathrm{TIR}}-L_{\mathrm{HCN}}^{\prime}$ are tight in the whole sample with 

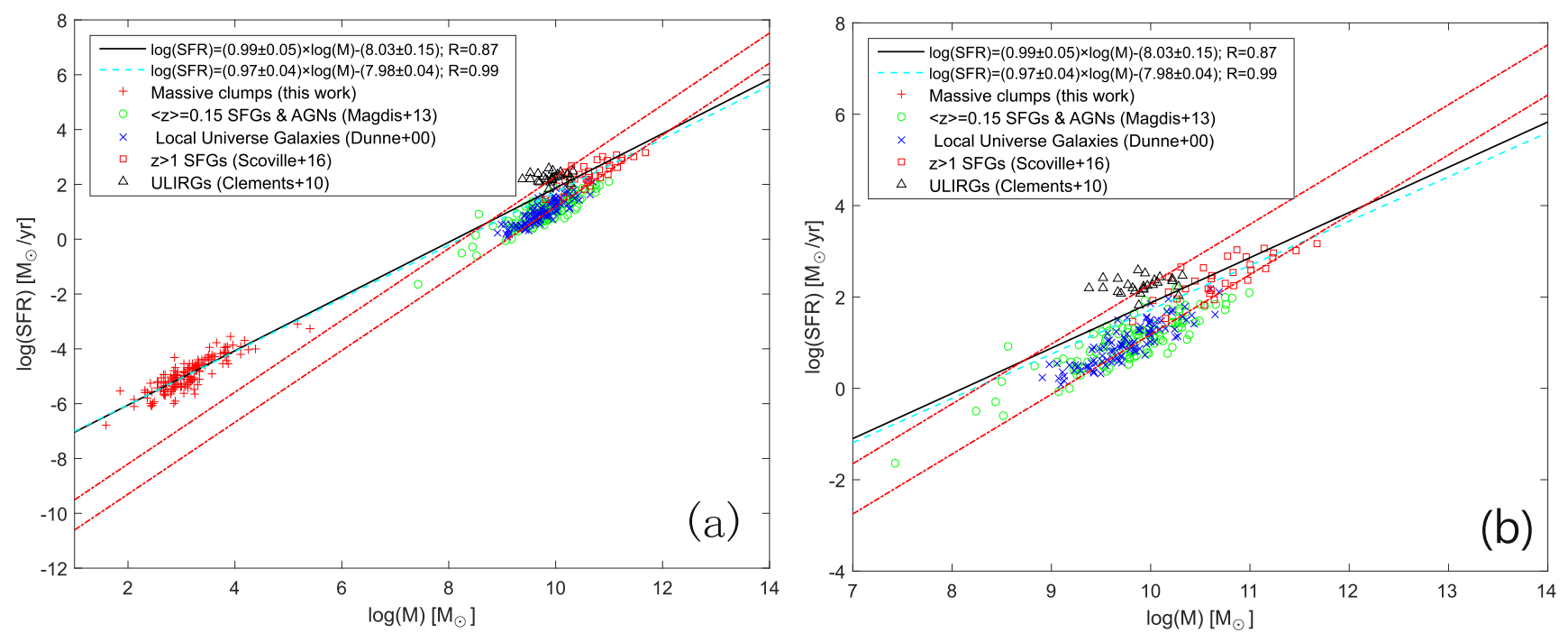

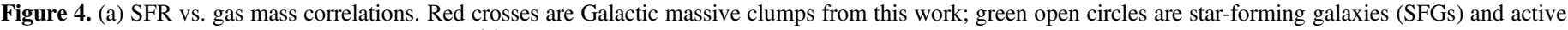

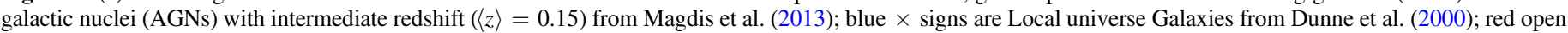

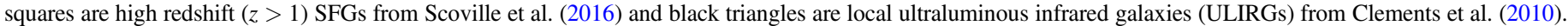

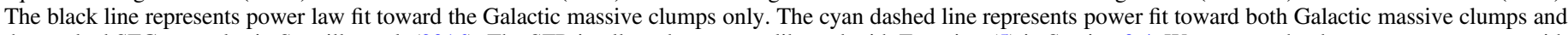

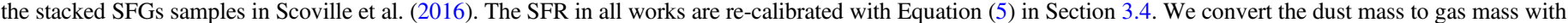

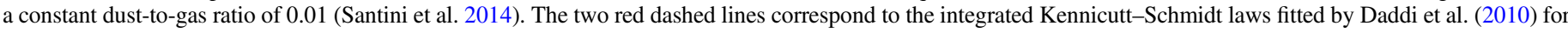

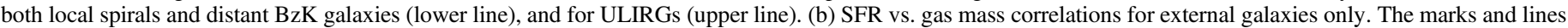
are the same as in panel (a).

$L_{\text {TIR }}$ down to at least $10^{3} L_{\odot}$. We also noticed that the sublinear $L_{\mathrm{TIR}}-L_{\mathrm{HCN}}^{\prime}$ correlation may even extend to the low luminosity $\left(L_{\mathrm{TIR}}<10^{2} L_{\odot}\right)$ end. However, we only have one data point with $L_{\mathrm{TIR}}<10^{3} L_{\odot}$ in the $L_{\mathrm{TIR}}-L_{\mathrm{HCN}}^{\prime}$ plot. More observations of low luminosity clumps would be helpful.

In panels (c) and (d) of Figure 5, we extend the $L_{\mathrm{TIR}}-L_{\mathrm{CS}}^{\prime}$ and $L_{\mathrm{TIR}}-L_{\mathrm{HCN}}^{\prime}$ correlations from Galactic molecular clumps to active galaxies. The data of active galaxies are from Zhang et al. (2014). We only include their data with strong detections $(\geqslant 4 \sigma)$. Both $L_{\mathrm{TIR}}-L_{\mathrm{CS}}^{\prime}$ and $L_{\mathrm{TIR}}-L_{\mathrm{HCN}}^{\prime}$ correlations are very tight with slopes close to unit over about ten orders of magnitude in $L_{\mathrm{TIR}}$. The best linear least-squares fits with uncertainties are listed below, with $R$ indicating the correlation coefficient:

$$
\begin{aligned}
\log \left(L_{\mathrm{TIR}}\right)= & (1.02 \pm 0.03) \times \log \left(L^{\prime}{ }_{\mathrm{CS}}\right) \\
& +(3.80 \pm 0.04) ; R=0.96 \\
\log \left(L_{\mathrm{TIR}}\right)= & (1.03 \pm 0.02) \times \log \left(L^{\prime}{ }_{\mathrm{HCN}}\right) \\
& +(3.58 \pm 0.04) ; R=0.98 .
\end{aligned}
$$

The slope (1.02 \pm 0.03$)$ of CS (7-6) derived here is consistent with the one $(1.00 \pm 0.01)$ in Zhang et al. (2014). From Bayesian regression with LINMIX_ERR (Kelly 2007), the slopes of $L_{\mathrm{TIR}}-L_{\mathrm{CS}}^{\prime}$ and $L_{\mathrm{TIR}}-L_{\mathrm{HCN}}^{\prime}$ are $1.05 \pm 0.02$ and $1.03 \pm 0.02$, respectively, which are consistent with results from linear least-squares fits. We noticed that the total IR luminosity in Zhang et al. (2014) was obtained by using the four IRAS bands equation as in other previous works (Gao \& Solomon 2004; Wu et al. 2005, 2010), rather than by integrating the SED as we did toward massive clumps. However, in Section 4.1, we will demonstrate that there is little difference between the total IR luminosity $\left(L_{\mathrm{IR}}\right)$ derived from IRAS four bands and the total IR luminosity $\left(L_{\mathrm{TIR}}\right)$ derived by integrating the SED. If we use $L_{\mathrm{IR}}$ instead of $L_{\mathrm{TIR}}$ for Galactic molecular clumps, the correlations for both Galactic molecular clumps and active galaxies in Figure 5 will become:

$$
\begin{aligned}
\log \left(L_{\mathrm{IR}}\right)= & (1.05 \pm 0.06) \times \log \left(L_{\mathrm{CS}}^{\prime}\right) \\
& +(3.85 \pm 0.04) ; R=0.97 \\
\log \left(L_{\mathrm{IR}}\right)= & (1.02 \pm 0.03) \times \log \left(L_{\mathrm{HCN}}^{\prime}\right) \\
& +(3.63 \pm 0.05) ; R=0.99 .
\end{aligned}
$$

These correlations are well consistent with those derived with $L_{\mathrm{TIR}}$.

\section{DISCUSSIONS}

\subsection{Caveats in Estimating Infrared Luminosity and Line Luminosity}

As shown in panel (a) of Figure 5, the correlation between infrared luminosity and CS (7-6) line luminosity derived for our sample is slightly different from Wu et al. (2010). Such difference comes from the different methods used to derive infrared luminosity and CS (7-6) line luminosity. In our work, we used the total infrared luminosity $\left(L_{\mathrm{TIR}}\right)$ from integrating the whole SED (Faúndez et al. 2004). While in $\mathrm{Wu}$ et al. $(2005,2010)$, the total infrared luminosity $\left(L_{\mathrm{IR}}\right.$ in $\left.L_{\odot}\right)$ was calculated based on the four IRAS bands:

$$
\begin{aligned}
& L_{\mathrm{IR}}=0.56 \times D^{2} \times\left(13.48 \times f_{12}+5.16\right. \\
& \left.\quad \times f_{25}+2.58 \times f_{60}+f_{100}\right),
\end{aligned}
$$

where $f_{x}$ is the flux in band $\mathrm{x}$ in units of $\mathrm{Jy}, D$ is distance in kpc. We also derived $L_{\mathrm{IR}}$ based on the four IRAS bands and present them in second column of Table 2. In panel (a) of Figure 7, we present the derived $L_{\mathrm{IR}}$ and $L_{\mathrm{TIR}} . L_{\mathrm{IR}}$ and $L_{\mathrm{TIR}}$ are nearly linearly correlated with each other as:

$$
\begin{aligned}
& \log \left(L_{\mathrm{IR}}\right)=(1.03 \pm 0.01) \times \log \left(L_{\mathrm{TIR}}\right) \\
& -(0.10 \pm 0.03) ; R=1.00 .
\end{aligned}
$$



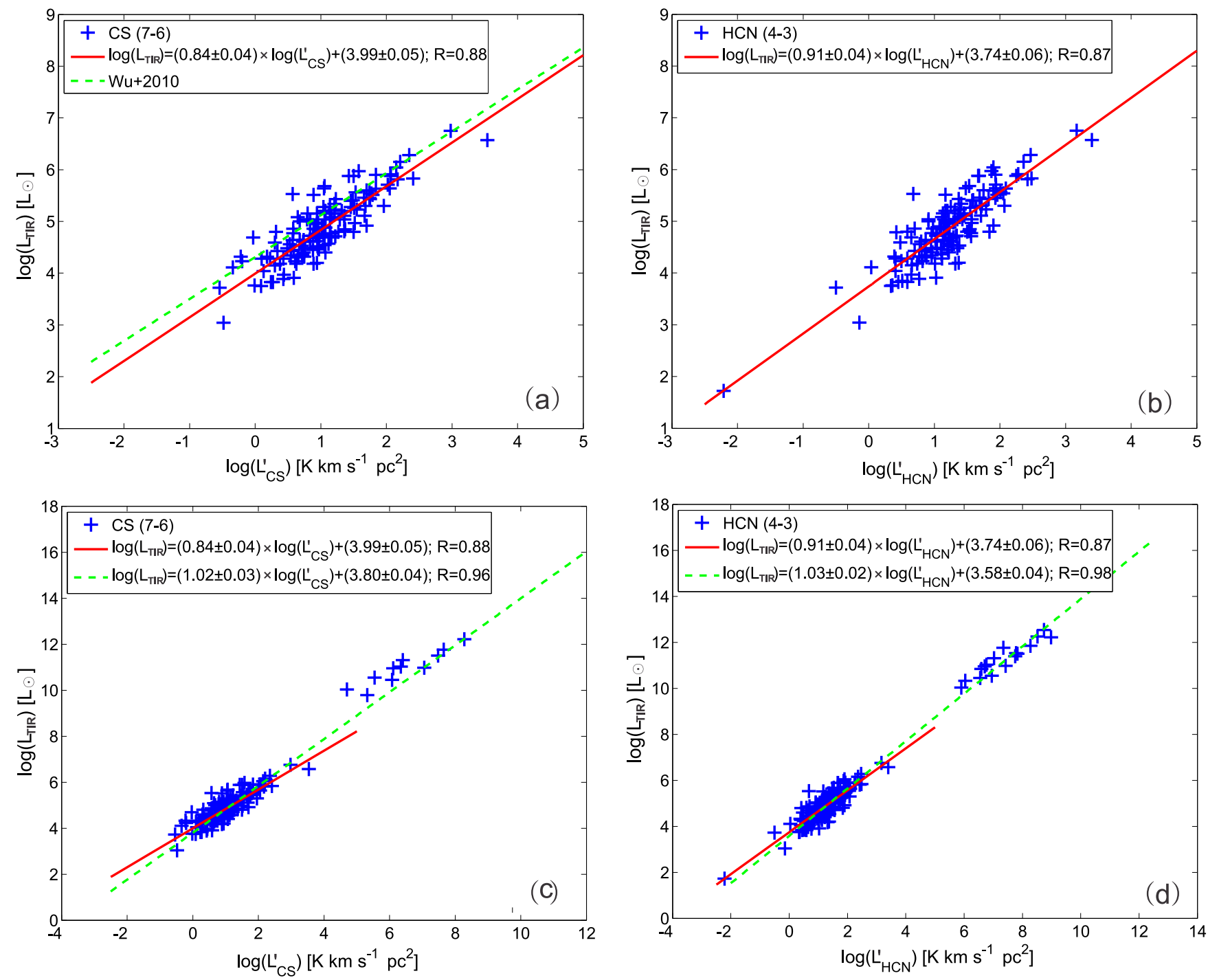

Figure 5. (a): $L_{\mathrm{TIR}}-L_{\mathrm{CS}}^{\prime}$ correlation for Galactic clumps. (b): $L_{\mathrm{TIR}}-L_{\mathrm{HCN}}^{\prime}$ correlation for Galactic clumps. (c): upper-left panel: $L_{\mathrm{TIR}}-L_{\mathrm{CS}}^{\prime}$ correlation for Galactic clumps and active galaxies. (d): $L_{\mathrm{TIR}}-L_{\mathrm{HCN}}^{\prime}$ correlation for Galactic clumps and active galaxies. The red lines in each panel represent least squares fits toward Galactic clumps. The green dashed line in the upper left panel represent the least squares fits toward Galactic clumps from Wu et al. (2010). The green dashed lines in the lower panels represent the least squares fits toward both Galactic clumps and active Galaxies.

In panel (b) of Figure 7, we investigate the relation between $L_{\mathrm{IR}}$ and $L_{\mathrm{CS}}^{\prime}$. The $L_{\mathrm{IR}}-L_{\mathrm{CS}}^{\prime}$ correlation for our sample is nearly the same as $L_{\mathrm{TIR}}-L_{\mathrm{CS}}^{\prime}$ correlation, indicating that different methods in deriving infrared luminosity should not affect the interpretation of the $\mathrm{K}-\mathrm{S}$ law.

The intercept for our sample is smaller than the one of $\mathrm{Wu}$ et al. (2010). It is probably because we used continuum size to calculate CS (7-6) line luminosity. Since lines are easily excited in subthermally populated gas with densities more than an order of magnitude lower than critical density $n_{\text {crit }}$, the effective excitation density $n_{\text {eff }}$, rather than $n_{\text {crit }}$, is more suitable to characterize the environment where a transition is excited (Reiter et al. 2011). The CS (7-6) has a much higher effective excitation density $\left(2.1 \times 10^{6} \mathrm{~cm}^{-3}\right.$ at $\left.20 \mathrm{~K}\right)$ than the median volume density $\left(\sim 1.0 \times 10^{5} \mathrm{~cm}^{-3}\right)$ of our sample. Therefore the emission size of CS (7-6) should be smaller than the continuum emission size.

Wu et al. (2010) mapped 52 clumps in CS (7-6). The 25 of these 52 clumps were also mapped in $350 \mu \mathrm{m}$ continuum emission by Mueller et al. (2002). The median ratio of CS (7-6) emission size $\left(\right.$ Size $\left._{\mathrm{cs}}\right)$ to the $350 \mu \mathrm{m}$ continuum emission size $\left(\right.$ Size $_{0.35 \mathrm{~mm}}$ ) is $\sim 0.87$. Most sources both in our sample and the sample of $\mathrm{Wu}$ et al. (2010) are UC H II regions. The median infrared luminosity in the sample of $\mathrm{Wu}$ et al. (2010) is $1.06 \times 10^{5} L_{\odot}$, which is comparable to the median infrared luminosity $\left(6.7 \times 10^{4} L_{\odot}\right)$ of our sample. Especially, the 114 most luminous clumps in our sample also has a median infrared luminosity of $1.1 \times 10^{5} L_{\odot}$. Therefore, we assume that the sources in our sample share similar properties as the sources in $\mathrm{Wu}$ et al. (2010). If we assume that the $1.2 \mathrm{~mm}$ continuum emission size ( $\operatorname{Size}_{1.2 \mathrm{~mm}}$ ) is similar to $\operatorname{Size}_{0.35 \mathrm{~mm}}$ and all the sources have a constant $\operatorname{Size}_{\mathrm{cs}} / \mathrm{Size}_{1.2 \mathrm{~mm}}$ ratio of 0.87, we can estimate the overestimation factor in CS (7-6) line luminosity. We admit that further mapping observations are needed to test these assumptions. The $1.2 \mathrm{~mm}$ continuum angular sizes range from $26^{\prime \prime}$ to $70^{\prime \prime}$ in our sample. Therefore, the corresponding CS (7-6) line luminosity derived in Section 3.2 should be overestimated by a factor of $\frac{\text { Size }_{1.2 \mathrm{~mm}}^{2}+22^{2}}{\left(\operatorname{Size}_{1.2 \mathrm{~mm}} \times 0.87\right)^{2}+22^{2}}-1$, which ranges from $17 \%$ to $28 \%$. The overestimation of CS (7-6) line luminosity leads to a smaller intercept of $L_{\mathrm{IR}}-L_{\mathrm{CS}}^{\prime}$ correlation. 

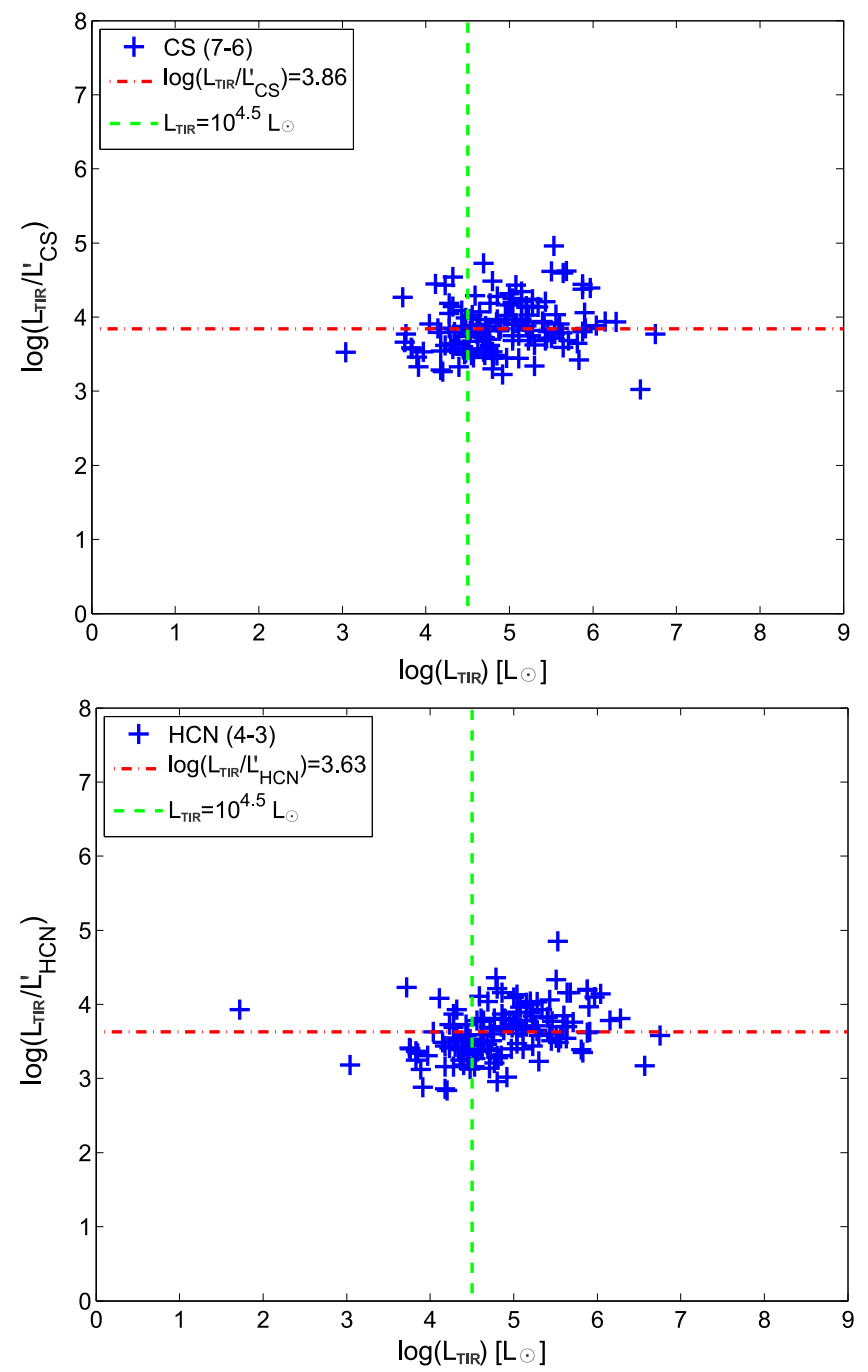

Figure 6. Correlations between $L_{\mathrm{TIR}} / L_{\text {mol }}^{\prime}$ and $L_{\mathrm{TIR}}$ for CS (7-6) (Upper panel) and HCN (4-3) (Lower panel). The horizontal dashed lines indicate the median $L_{\mathrm{TIR}} / L_{\text {mol }}^{\prime}$ ratio. The vertical dashed lines show the cutoff at $\mathrm{L}_{\mathrm{TIR}}=10^{4.5} L_{\odot}$, suggested in Wu et al. $(2005,2010)$.

However, since the factor of overestimation for sources with different angular sizes only varies by $<11 \%$, the slopes of correlations should not be severely affected by size assumption. Indeed, as shown in panel (a) of Figure 5, the slope of $L_{\mathrm{IR}}-L_{\mathrm{CS}}^{\prime}$ correlation derived from our single pointing observations is very consistent with the slope derived from the mapping observations in Wu et al. (2010).

$\mathrm{Wu}$ et al. (2010) only fitted their data for clumps with $L_{\mathrm{IR}}>10^{4.5} L_{\odot}$. The best linear least-squares fit of $L_{\mathrm{IR}}-L_{\mathrm{CS}}^{\prime}$ for all the data of $\mathrm{Wu}$ et al. (2010) is:

$$
\begin{aligned}
\log \left(L_{\mathrm{IR}}\right)= & (0.82 \pm 0.10) \times \log \left(L^{\prime}{ }_{\mathrm{CS}}\right) \\
& +(4.23 \pm 0.13) ; R=0.79
\end{aligned}
$$

The best linear least-squares fit of $L_{\mathrm{IR}}-L_{\mathrm{CS}}^{\prime}$ for our sample is:

$$
\begin{aligned}
\log \left(L_{\mathrm{IR}}\right)= & (0.85 \pm 0.04) \times \log \left(L_{\mathrm{CS}}^{\prime}\right) \\
& +(4.01 \pm 0.06) ; R=0.87
\end{aligned}
$$

The best linear least-squares fit of $L_{\mathrm{IR}}-L^{\prime}{ }_{\mathrm{CS}}$ for both our sample and the sample of $\mathrm{Wu}$ et al. (2010) is:

$$
\begin{aligned}
\log \left(L_{\mathrm{IR}}\right)= & (0.84 \pm 0.04) \times \log \left(L^{\prime}{ }_{\mathrm{CS}}\right) \\
& +(4.04 \pm 0.05) ; R=0.86
\end{aligned}
$$

The slopes of these three correlations are consistent with each other considering errors, strongly indicating that the slopes are not affected by the assuming of the same size between dense gas and dust. We noticed that the effective excitation density $\left(2.4 \times 10^{5} \mathrm{~cm}^{-3}\right.$ at $\left.20 \mathrm{~K}\right)$ of $\mathrm{HCN}(4-3)$ is comparable to the median volume density of our sample, indicating that the $\mathrm{HCN}$ (4-3) line luminosity should not be overestimated as severely as CS (7-6).

To further investigate the effect of size assumption on $L_{\mathrm{TIR}}{ }^{-}$ $L_{\text {mol }}^{\prime}$ correlations, we derive a lower limit for line luminosity with Equation (1) assuming that the source size is much smaller than the beam size $\left(\theta_{\mathrm{s}} \ll \theta_{\text {beam }}\right)$. In panels (c) and (d) of Figure 7 , we investigate the correlations between infrared luminosity and line luminosity derived under such point source assumption for HCN (4-3) and CS (7-6), respectively. We find that the correlations have similar slopes and slightly larger intercepts when compared with the values derived by assuming a source size the same as continuum emission size. The slope $(0.80 \pm 0.04)$ for CS (7-6) is consistent with the value of $\mathrm{Wu}$ et al. (2010). But the intercept $(4.49 \pm 0.04)$ for CS (7-6) is slightly larger than the value of Wu et al. (2010). Therefore, the line luminosities obtained in Section 3.2 should be systematically overestimated especially for CS (7-6). However, the slopes of $L_{\mathrm{TIR}}-L_{\text {mol }}^{\prime}$ correlations are not affected as much as intercepts.

In conclusion, our single pointing results are consistent with previous mapping results considering the uncertainties. The slopes of $L_{\mathrm{TIR}}-L_{\text {mol }}^{\prime}$ correlations are not affected by uncertainties in measurements of $L_{\mathrm{TIR}}$ and $L_{\text {mol }}^{\prime}$.

\subsection{Dependance of Dense Molecular Gas Star Formation Law on Physical Parameters}

In Figures 8 and 9, we investigate how the dense molecular gas star formation law depends on different physical parameters, such as volume density, dust temperature, luminosityto-mass ratio, $\sigma_{\text {line }} / \sigma_{\text {vir }}$, and line profiles for Galactic clumps. Taking "volume density" for example, we first sorted the clumps according to their volume density values. Then we investigated the $L_{\mathrm{TIR}}-L_{\mathrm{CS}}^{\prime}$ and $L_{\mathrm{TIR}}-L_{\mathrm{HCN}}^{\prime}$ correlations toward the sources with volume densities ranked in the lower third and upper third, respectively. The median values of volume density in the lower third and the upper third groups are $3.7 \times 10^{4} \mathrm{~cm}^{-3}$ and $2.8 \times 10^{5} \mathrm{~cm}^{-3}$, respectively. As shown in panels (a) and (b) in Figure 8, the $L_{\mathrm{TIR}}-L_{\mathrm{CS}}^{\prime}$ and $L_{\mathrm{TIR}}-L_{\mathrm{HCN}}^{\prime}$ correlations seem not to depend on volume density. Especially for HCN (4-3), correlations of both low density and high density clumps are very close to linear. We notice that the volume density is anti-correlated with distance due to the different effective linear resolution. The determinations of some quantities, such as surface and volume densities for distant clumps, could be biased to lower values due to worse effective linear resolution (Wu et al. 2010). Therefore, the fact that $L_{\mathrm{TIR}}-L_{\text {mol }}^{\prime}$ correlation does not change with volume density does not mean that star formation efficiencies (SFEs) are not affected by density. In other words, $L_{\mathrm{TIR}}-L_{\text {mol }}^{\prime}$ correlation is not biased by distance. 

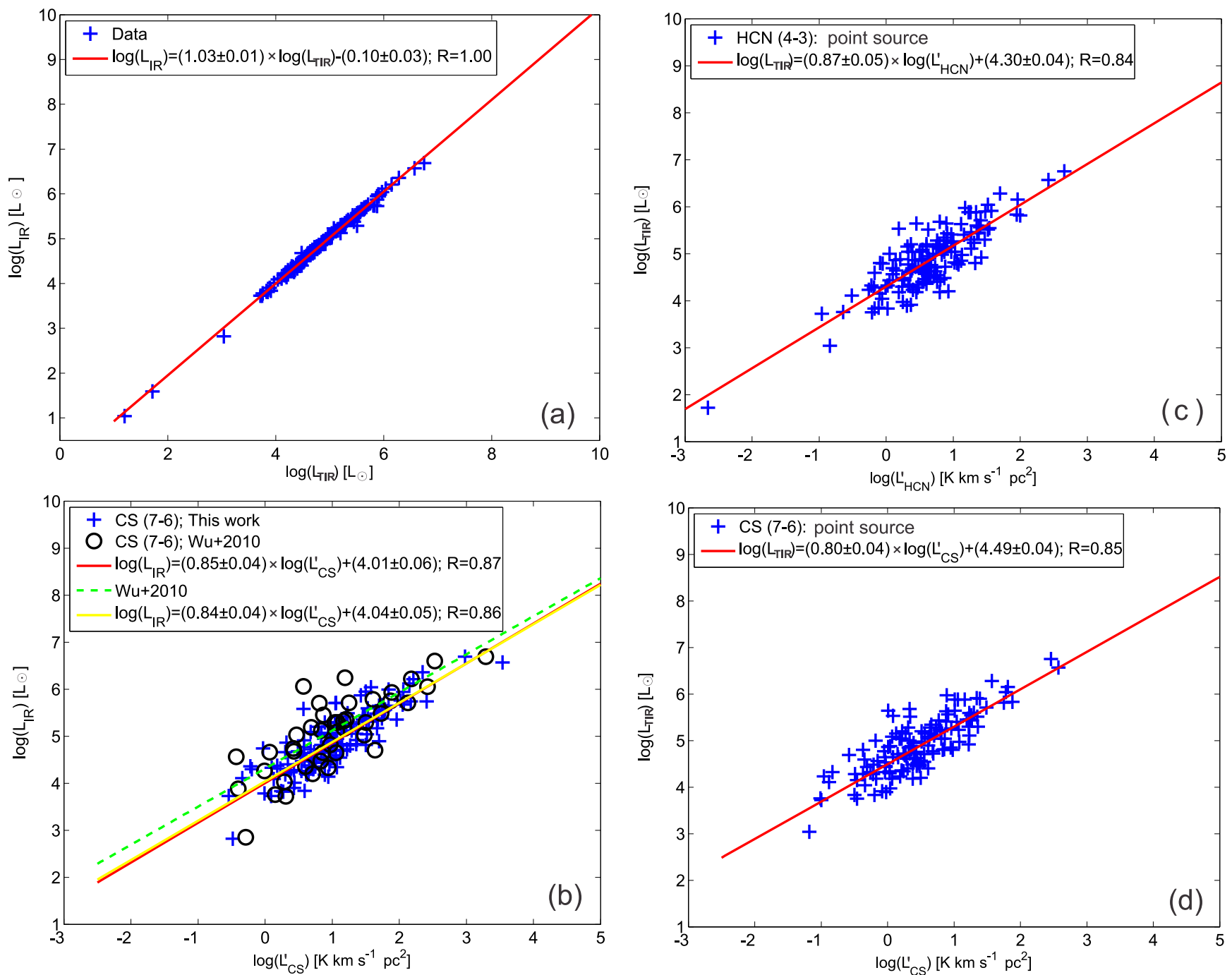

Figure 7. (a): correlation between IR luminosities $\left(L_{\mathrm{IR}}\right)$ derived from IRAS four bands and IR luminosities $\left(L_{\mathrm{TIR}}\right)$ derived from integrating whole SED. (b): comparison of $L_{\mathrm{IR}}-L_{\mathrm{CS}}^{\prime}$ correlations in our sample with correlations in the sample of Wu et al. (2010). (c): correlation between $L_{\mathrm{TIR}}$ and beam-averaged $L_{\mathrm{HCN}}^{\prime}$. (d): correlation between $L_{\mathrm{TIR}}$ and beam-averaged $L_{\mathrm{CS}}^{\prime}$.

We also sorted the sample according to values of other physical parameters (dust temperature, luminosity-to-mass ratio, $\sigma_{\text {line }} / \sigma_{\text {vir }}$, and then investigated the $L_{\mathrm{TIR}}-L_{\mathrm{CS}}^{\prime}$ and $L_{\mathrm{TIR}}-L_{\mathrm{HCN}}^{\prime}$ correlations in the lower third and the upper third groups. The slopes $(\alpha)$, intercepts $(\beta)$ and correlation coefficients $(R)$ of the correlations in each group are summarized in Table 3. In the first column of Table 3, we present the median values of the physical parameters of each group. Interestingly, we find that $L_{\mathrm{TIR}}-L_{\mathrm{CS}}^{\prime}$ and $L_{\mathrm{TIR}}-L_{\mathrm{HCN}}^{\prime}$ correlations show significant changes according to different dust temperature, luminosity-to-mass ratio and $\sigma_{\text {line }} / \sigma_{\text {vir }}$. The $L_{\mathrm{TIR}}-L_{\text {mol }}^{\prime}$ correlations show clear bimodal behavior. The correlations of clumps with lower dust temperature, lower luminosity-to-mass ratio and higher $\sigma_{\text {line }} / \sigma_{\text {vir }}$ are closer to linear. While the correlations of clumps with higher dust temperature, higher luminosity-to-mass ratio and lower $\sigma_{\text {line }} /$ $\sigma_{\text {vir }}$ tend toward sublinear. It seems that sources with higher temperature and luminosity-to-mass ratios have higher $\frac{L_{T R}}{L_{\text {mol }}^{\prime}}$. Sources with smaller $\sigma_{\text {line }} / \sigma_{\text {vir }}$ ratios, which are more likely gravitationally bound, have higher $\frac{L_{\mathrm{TIR}}}{L_{\mathrm{mol}}}$. Those sources with higher $\sigma_{\text {line }} / \sigma_{\text {vir }}$ ratios are more turbulent and probably are more affected by stellar feedback (like outflows), indicating that turbulence and stellar feedback could greatly reduce SFEs in molecular clouds, leading to a smaller $\frac{L_{\mathrm{TRR}}}{L^{\prime} \mathrm{mal}}$.

More evolved sources have higher infrared luminosities and probably have also consumed more gas, leading to higher luminosity-to-mass ratios (Ma et al. 2013). The luminosity-tomass ratio should be a good evolutionary tracer (Liu et al. 2013; Ma et al. 2013). Therefore, the bimodal behavior of star formation law described above is more likely due to evolutionary effect. The sources in our sample were selected with IR colors similar to UC H II regions. They represent massive molecular clumps with embedded massive star formation. In contrast, massive infrared dark clouds have very low luminosity-to-mass ratios since they have not yet formed massive stars. Furthermore, classical HII regions are luminous, but have very little associated molecular gas. Therefore, for sources at different evolutionary stages, the current (or observed) SFEs probed by $L_{\mathrm{IR}} / M_{\text {clump }}$ or $L_{\mathrm{IR}} / L_{\text {mol }}^{\prime}$ should be very diverse at clump scale as also suggested by Stephens et al. (2016). Our results in Figure 8 indicate that the slopes of $L_{\mathrm{TIR}^{-}}$ $L_{\text {mol }}^{\prime}$ correlations (or current star formation law) become more shallow as clumps evolve. 

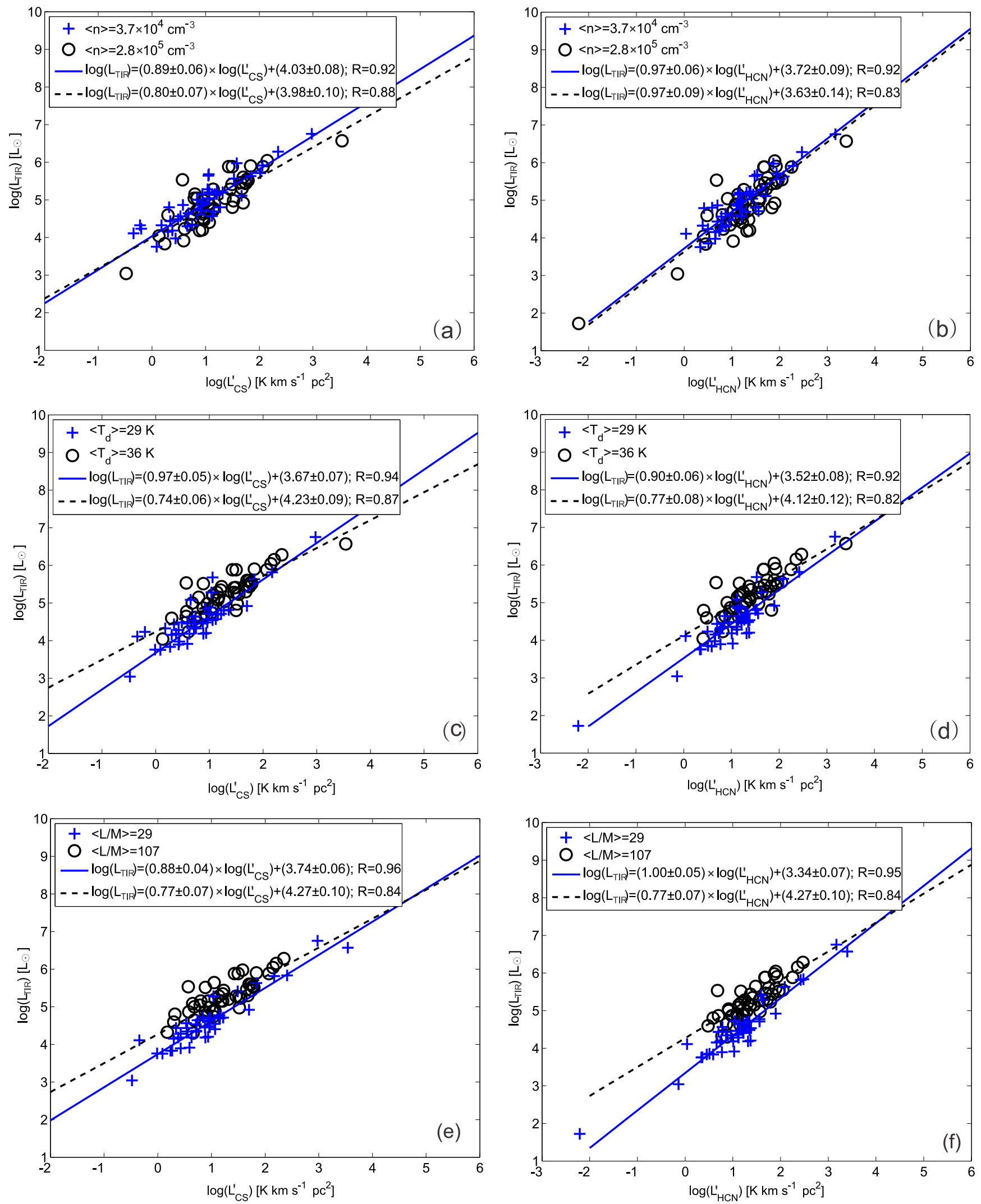

Figure 8. (a): $L_{\mathrm{TIR}}-L_{\mathrm{CS}}^{\prime}$ correlations for clumps with smallest volume densities (blue crosses) and for clumps with largest volume densities (open circles). (b): $L_{\mathrm{TIR}}{ }^{-}$ $L_{\mathrm{HCN}}^{\prime}$ correlations for clumps with smallest volume densities (blue crosses) and for clumps with largest volume densities (open circles). (c): $L_{\mathrm{TIR}}-L_{\mathrm{CS}}^{\prime}$ correlations for clumps with smallest dust temperature (blue crosses) and for clumps with largest dust temperature (open circles). (d): $L_{\mathrm{TIR}}-L_{\mathrm{HCN}}^{\prime}$ correlations for clumps with smallest dust temperature (blue crosses) and for clumps with largest dust temperature (open circles). (e): $L_{\mathrm{TIR}}-L_{\mathrm{CS}}^{\prime}$ correlations for clumps with smallest luminosity-to-mass ratios (blue crosses) and for clumps with largest luminosity-to-mass ratios (open circles). (f): $L_{\mathrm{TIR}}-L_{\mathrm{HCN}}^{\prime}$ correlations for clumps with smallest luminosity-to-mass ratios (blue crosses) and for clumps with largest luminosity-to-mass ratios (open circles). 

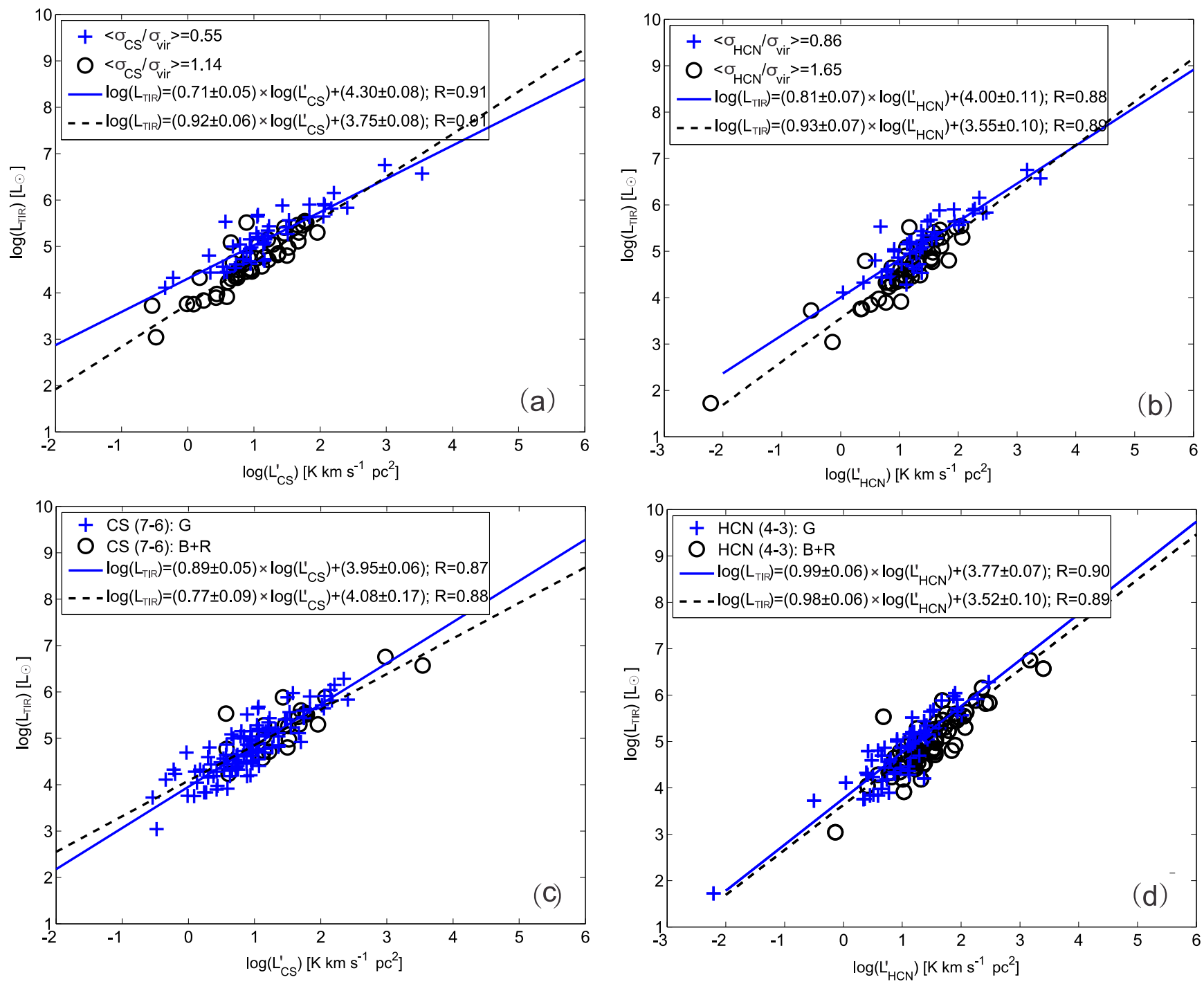

Figure 9. (a): $L_{\mathrm{TIR}}-L_{\mathrm{CS}}^{\prime}$ correlations for clumps with smallest $\sigma_{\mathrm{CS}} / \sigma_{\mathrm{vir}}$ (blue crosses) and for clumps with largest $\sigma_{\mathrm{CS}} / \sigma_{\mathrm{vir}}$ (open circles). (b): $L_{\mathrm{TIR}}-L_{\mathrm{HCN}}^{\prime}$ correlations for clumps with smallest $\sigma_{\mathrm{HCN}} / \sigma_{\mathrm{vir}}$ (blue crosses) and for clumps with largest $\sigma_{\mathrm{HCN}} / \sigma_{\mathrm{vir}}$ (open circles). (c): $L_{\mathrm{TIR}}-L_{\mathrm{CS}}^{\prime}$ correlations for clumps with symmetric profiles (blue crosses) and for clumps with asymmetric profiles (open circles). (d): $L_{\mathrm{TIR}}-L_{\mathrm{HCN}}^{\prime}$ correlations for clumps with symmetric profiles (blue crosses) and for clumps with asymmetric profiles (open circles).

Table 3

Parameters of $L_{\mathrm{TIR}}-L_{\text {mol }}^{\prime}$ Correlations for Each Sub-sample

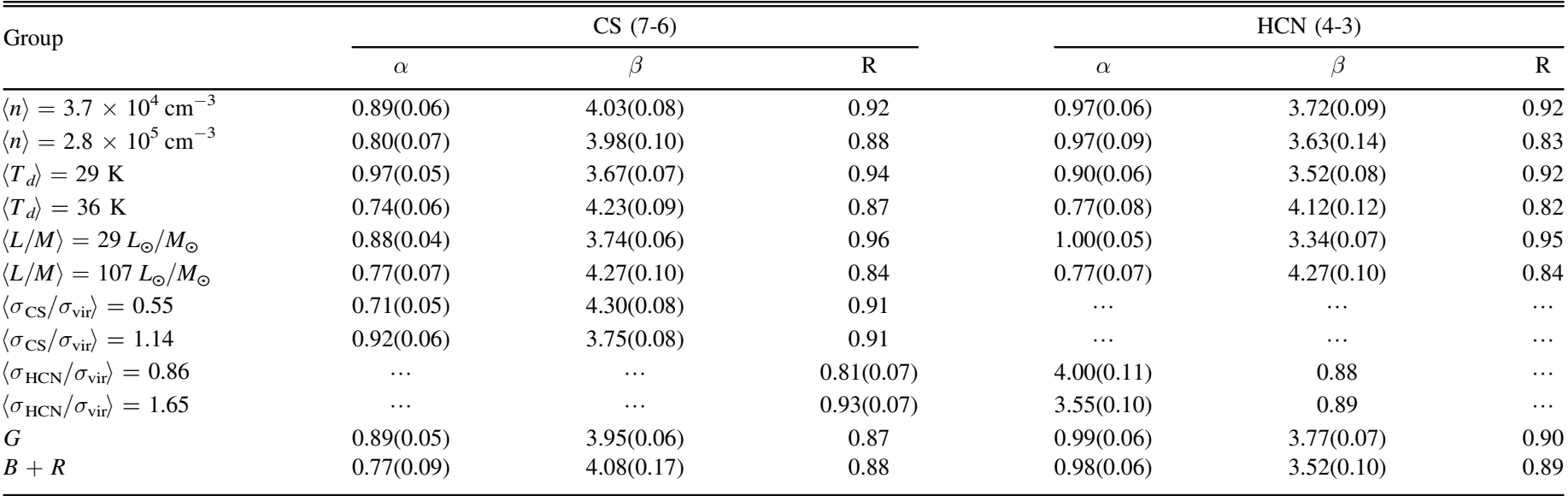


Is the bimodal behavior found in the $L_{\mathrm{TIR}}-L_{\text {mol }}^{\prime}$ correlations affected by the assuming of the same size between dense gas $\left(\right.$ size $\left._{\mathrm{mol}}\right)$ and dust ( size $\left._{\text {dust }}\right)$. More evolved (higher $L / M$ ) sources likely also have higher temperature, which could be more efficient to excite the dense gas tracer lines comparing to the cooler sources in the earlier stages (Molinari et al. 2016). So the late stage sources might have higher $\operatorname{size}_{\text {mol }} /$ size $_{\text {dust }}$ ratios than the sources in the early stages. We investigate this effect with $\mathrm{HCN}$ (4-3). In extreme case, the line luminosity of a point source will be overestimated by a factor of $\left(35^{2}+22^{2}\right) /$ $22^{2}=3.5$ if we take the median continuum size of $35^{\prime \prime}$ as the line emission size. If all the sources in the low $L / M$ $(\langle L / M\rangle=29)$ subsample are point source like in $\mathrm{HCN}(4-3)$ emission, the HCN (4-3) line luminosity in panel (f) of Figure 8 should be systematically overestimated by a factor of $\sim 3.5$ and the corresponding intercept of $L_{\mathrm{TIR}}-L_{\text {mol }}^{\prime}$ should be underestimated by $\sim 0.55$. However, even in this extreme case, the intercept ( 3.89) of the lowL/M $(\langle L / M\rangle=29$ subsample is still lower than that $(\sim 4.27)$ of the high $L / M(\langle L / M\rangle=107$ subsample. Therefore, we argue that the bimodal behavior in the $L_{\mathrm{TIR}}-L_{\text {mol }}^{\prime}$ correlations is not greatly affected by size assumption. Future mapping observations will test this hypotheses.

In panels (c) and (d) of Figure 9, we investigate the $L_{\mathrm{TIR}}-$ $L_{\mathrm{CS}}^{\prime}$ and $L_{\mathrm{TIR}}-L_{\mathrm{HCN}}^{\prime}$ correlations for sources with symmetric line profiles (" $G$ ") and asymmetric line profiles (" $B+R$ "). In general, considering the errors, the correlations for sources with different line profiles do not show significant differences.

\subsection{Comparison Between Different Tracers}

In Figure 10, we present the correlations between clump masses and line luminosities for HCN (4-3) and CS (7-6). The $M_{\text {clump }}-L_{\text {CS }}^{\prime}$ and $M_{\text {clump }}-L_{\text {HCN }}^{\prime}$ correlations are very tight and sublinear. For comparison, we also investigate the correlations between clump masses and line luminosities for $J=2-1$, $J=5-4, J=7-6$ of CS and $J=1-0, J=3-2$ of HCN. The line luminosities are from $\mathrm{Wu}$ et al. (2010). The clump masses were calculated with $350 \mu \mathrm{m}$ continuum emission (Mueller et al. 2002). As shown in Figure 11, the clump masses are also strongly correlated with line luminosities of these transitions. The slopes $(\alpha)$, intercepts $(\beta)$ and correlation coefficients $(R)$ of the correlations were summarized in Table 4. For CS (7-6), the correlations for the sample of Wu et al. (2010) and our sample are consistent with similar slopes. In general, considering the errors, the correlations for lower transitions like $J=2-1$, $J=5-4$ of CS and $J=1-0, J=3-2$ of $\mathrm{HCN}$ are close to linear. While for the other higher transitions, the correlations are sublinear.

In Table 4 , we also present the parameters of the $L_{\mathrm{TIR}}-L_{\text {mol }}^{\prime}$ correlations. The parameters of the $L_{\mathrm{TIR}}-L_{\mathrm{mol}}^{\prime}$ correlations for $J=2-1, J=5-4$ of CS and $J=1-0, J=3-2$ of HCN were taken from $\mathrm{Wu}$ et al. (2010). It should be noted that $\mathrm{Wu}$ et al. (2010) only fitted the correlations for clumps with infrared luminosities larger than $10^{4.5} L_{\odot}$, where the initial mass function is fully sampled. Therefore, to compare with their results, we also fitted the $L_{\mathrm{TIR}}-L^{\prime}{ }_{\text {mol }}$ correlations of CS (7-6) and HCN (4-3) for clumps with infrared luminosities larger than $10^{4.5} L_{\odot}$ in our sample. The derived slopes of CS (7-6) and $\mathrm{HCN}$ (4-3) are 0.72(0.05) and 0.74(0.06), respectively, which are smaller than the corresponding slopes for all clumps with infrared luminosities larger than $10^{3} \mathrm{~L}_{\odot}$. In general, as shown in Table 4 , the $L_{\mathrm{TIR}}-L_{\text {mol }}^{\prime}$ correlations steepen for line transitions with lower upper energies and lower effective excitation densities. Especially for $\mathrm{HCN}$ (4-3) and CS (7-6), their $L_{\mathrm{TIR}^{-}}$ $L_{\text {mol }}^{\prime}$ correlations are apparently sublinear. Narayanan et al. (2008) predicted similar trend for external galaxies. However, the slopes of HCN transitions at clump scale (this work) or at galaxy scale (Zhang et al. 2014) are all much higher than those predicted by Narayanan et al. (2008). Narayanan et al. (2008) argued that lines with high critical densities $\left(n_{\text {crit }}\right)$ greater than the mean density of most of the emitting clouds in a galaxy will have only a small amount of thermalized gas, leading to shallow slopes for their SFR $-L_{\text {mol }}^{\prime}$ (or $L_{\mathrm{TIR}}-L_{\text {mol }}^{\prime}$ ) correlations. However, lines can easily excited in subthermally populated gas with densities more than an order of magnitude lower than $n_{\text {crit }}$ (Reiter et al. 2011). In addition, $L_{\mathrm{TIR}}-L_{\text {mol }}^{\prime}$ correlations do not seem to depend on densities as shown in panels (a) and (b) of Figure 8. Therefore, Narayanan et al. (2008) may underestimated the line luminosities for high critical density tracers, leading to the shallow slopes in corresponding SFR $-L_{\text {mol }}^{\prime}$ correlations.

Lada et al. (2010) found that the SFR in molecular clouds is linearly proportional to the cloud mass above a gas volume density threshold of $\sim 10^{4} \mathrm{~cm}^{-3}$. We define the dense gas above this volume density threshold as star forming gas, which are directly related to star formation. Due to variations of the atmosphere mimic emission from extended astronomical objects, the maps obtained by ground-based bolometer arrays intrinsically filter the large-scale diffuse gas and are most sensitive to the highest column densities (Csengeri et al. 2016). Since diffuse gas is mainly distributed on the surface of dense clumps and is more likely to be filtered in bolometer observations, the continuum emission obtained by groundbased bolometer arrays is sensitive not only to high column density gas but also to high volume density gas if there are no overlapped clumps along the line of sight. In our sample, all the sources have single velocity component, indicating that there are no overlapped clumps along the same line of sight. The $1.1 \mathrm{~mm}$ continuum indeed traces high volume density (median value of $\sim 1 \times 10^{5} \mathrm{~cm}^{-3}$ ) star forming gas. The continuum emission obtained by ground-based bolometer arrays is also often used to trace dense gas in other Galactic studies (Wienen et al. 2015; Csengeri et al. 2016). In addition, the linear $L_{\mathrm{TIR}^{-}}$ $M_{\text {clump }}$ correlations in Figure 3 and panel (f) of Figure 11 also suggest that dust emission be one of the best mass tracers in star forming clumps.

For $\mathrm{HCN}$ (4-3) and CS (7-6), the $L_{\mathrm{TIR}}-L_{\text {mol }}^{\prime}$ correlations for clumps with infrared luminosities larger than $10^{4.5} L_{\odot}$ are apparently sublinear. What is the origin of such sublinear slopes? Since their upper energies and effective densities are much larger than the median dust temperature $(\sim 30 \mathrm{~K})$ and median volume density $\left(1 \times 10^{5} \mathrm{~cm}^{-3}\right)$ of the whole sample, high effective excitation density tracers like HCN (4-3) and CS (7-6) may only trace the densest and warmest portions of star forming gas in clumps. It seems that high effective excitation density tracers cannot linearly trace the total masses of star forming gas, leading to a sublinear correlations for both $M_{\text {clump }}-L_{\text {mol }}^{\prime}$ and $L_{\mathrm{TIR}}-L_{\text {mol }}^{\prime}$ relations. The linear correlations of $L_{\mathrm{TIR}}-L_{\mathrm{CS}}^{\prime}$ and $L_{\mathrm{TIR}}-L_{\mathrm{HCN}}^{\prime}$ for both Galactic clumps and external galaxies presented in panels (c) and (d) of Figure 5 may be artificial caused by the large dynamical range of the data. Since $L_{\mathrm{TIR}}$ is linearly correlated with $M_{\text {clump }}$, linear $M_{\text {clump }}-L_{\text {mol }}^{\prime}$ correlations for low effective excitation density tracers will naturally lead to linear $L_{\mathrm{TIR}}-L_{\text {mol }}^{\prime}$ correlations. The different 

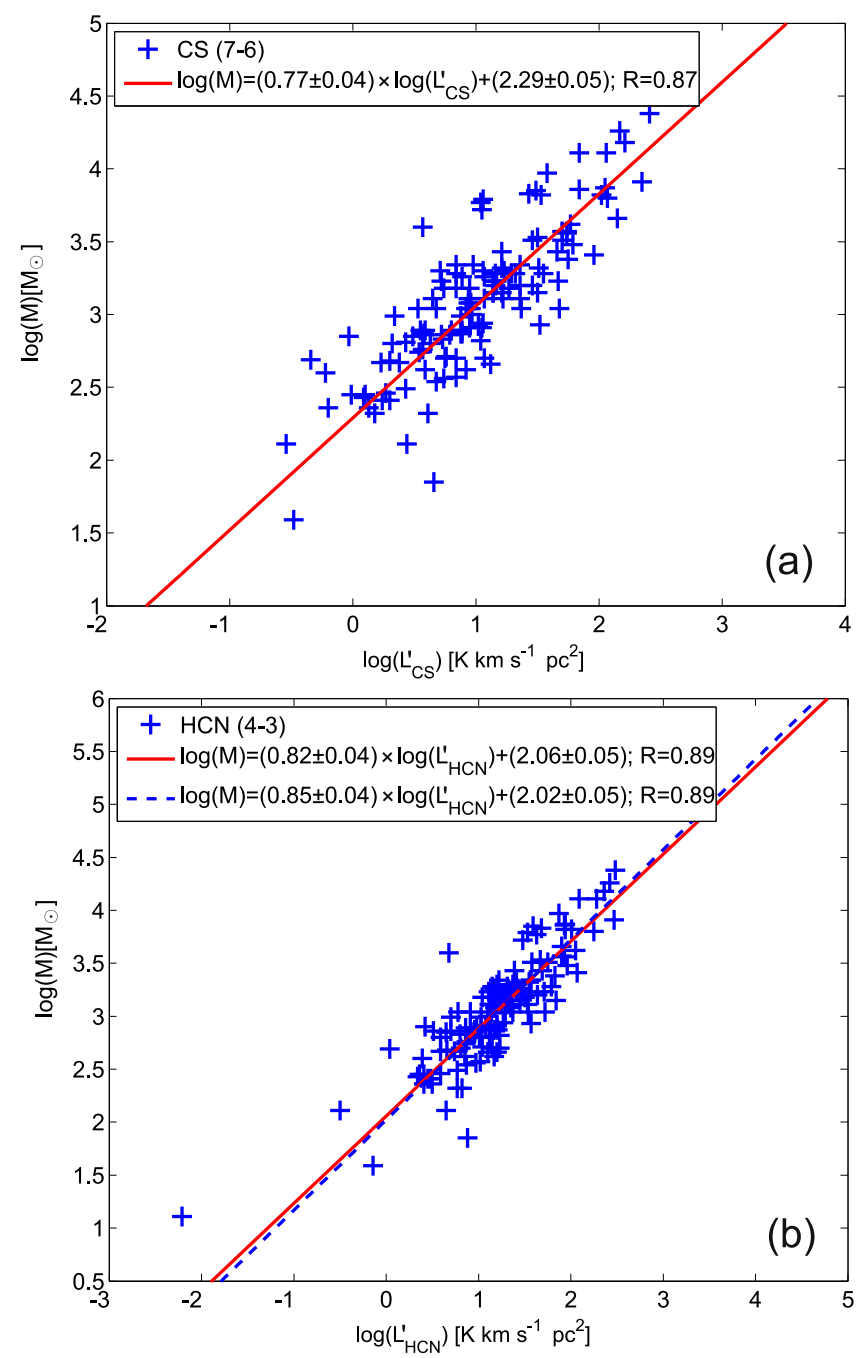

Figure 10. (a): clump masses vs. luminosities of CS (7-6). (b): clump masses vs. luminosities of HCN (4-3). The red line represent least squares fits for the whole sample. The blue line represents least squares fits for clumps with infrared luminosities larger than $10^{3} L_{\odot}$.

slopes of $\mathrm{K}-\mathrm{S}$ law for different molecular tracers are mainly determined by their excitation conditions. There should be no more underlying physics for different slopes indicated by various tracers.

We noticed that $\mathrm{Wu}$ et al. (2010) found nearly linear correlations between the dense gas luminosity $\left(L_{\text {mol }}^{\prime}\right)$ and the virial mass $\left(M_{\mathrm{vir}}\right)$ even for high effective excitation density tracers like CS (7-6). Their results seem to differ from ours. However, Wu et al. (2010) derived the virial masses for the gas traced by CS (7-6) by using the linewidths of $C^{34} S$ (5-4) lines and clump radii from CS (7-6) maps. However, since the high effective excitation density tracers like CS (7-6) and $C^{34} S(5-4)$ only can trace the densest and warmest portions of dense gas in clumps, the virial masses derived from these lines cannot represent the total masses of all the star forming gas. If they use total clump mass instead of $M_{\mathrm{vir}}$, the corresponding $L_{\mathrm{mol}}^{\prime}-M$ should be sublinear for high effective excitation density tracers like CS (7-6) as shown in panel (c) of Figure 11. Low effective excitation density tracers can better trace the total star forming gas. As shown in panel (a) of Figure 12, the virial masses derived from $\mathrm{HCN}$ (3-2) is linearly correlated with clump masses derived from dust continuum emission, which can explain the linear correlation of $M_{\text {clump }}-L_{\text {HCN }}^{\prime}$ in panel (e) of Figure 11. We derived the virial masses for the sources in our sample with the Equation 4 in $\mathrm{Wu}$ et al. (2010). We used the line widths of CS (7-6) and clump radii derived from $1.2 \mathrm{~mm}$ continuum. Interestingly, as shown in panel (b) of Figure 12, the clump masses derived from $1.2 \mathrm{~mm}$ continuum are linearly correlated with the virial masses, indicating that the $1.2 \mathrm{~mm}$ continuum emission well traces the bounded star forming gas. However, if we use the linewidths of optically thin lines (e.g., $\left.\mathrm{C}^{34} \mathrm{~S}(5-4)\right)$ and the radii of actual CS (7-6) emission area as Wu et al. (2010) did, the corresponding virial masses should be smaller than the total clump masses. The use of virial mass is based on virial equilibrium. However, the status of massive clumps may deviate from virial equilibrium if they are affected by bulk motions like energetic outflows or infall. Due to the differences of excitation conditions, virial masses derived from different molecular transitions may vary very much $(\mathrm{Wu}$ et al. 2010). Therefore, we suggest dust continuum emission be a better tracer of the total star forming gas than virial masses.

\subsection{Connect Star Formation Laws in Galactic Clumps to External Galaxies}

$\mathrm{Wu}$ et al. (2005) first tried to connect star formation laws in Galactic clumps to external galaxies. They found nearly linear $L_{\mathrm{IR}}-L_{\text {mol }}^{\prime}$ correlations for $\mathrm{HCN}$ (1-0) toward both Galactic dense clumps and galaxies. Previous works (Wu et al. 2010; Zhang et al. 2014) and this work for other dense gas tracers also revealed linear $L_{\mathrm{IR}}-L_{\text {mol }}^{\prime}$ correlations when connecting Galactic dense clumps to external galaxies. Wu et al. (2005) argued that such linear correlations indicate a constant SFR per unit mass from the scale of dense clumps to that of distant galaxies (Wu et al. 2005, 2010). However, in extragalactic studies, one cannot easily resolve individual molecular clumps and distinguish between clouds at various evolutionary stages. As discussed in Section 4.2, the current (or observed) SFEs probed by $L_{\mathrm{IR}} / M_{\text {clump }}$ or $L_{\mathrm{IR}} / L_{\text {mol }}^{\prime}$ for clumps at different evolutionary stages are very diverse and the slopes of $L_{\mathrm{TIR}}{ }^{-}$ $L_{\text {mol }}^{\prime}$ correlations become more shallow as clumps evolve. The global star formation law inferred in extragalactic studies is a mixture of the star formation laws of different stages of star forming clumps/regions. Therefore, the linear $L_{\mathrm{IR}}-L_{\mathrm{mol}}^{\prime}$ correlations may only indicate a constant SFR per unit mass (or $L_{\mathrm{IR}} / L_{\text {mol }}^{\prime}$ ) on scales much larger than clump scale. Stephens et al. (2016) suggested that $L_{\mathrm{IR}} / L_{\text {mol }}^{\prime}$ may become constant at some scales larger than $\sim 1 \mathrm{kpc}$ because at only this size-scale will contain clumps to completely sample the clump mass function (CMF) and IMF.

In extragalactic studies, the $\mathrm{K}-\mathrm{S}$ scaling relations seem to change with redshifts, indicating that the star formation efficiency (or gas depletion time) also evolves with redshifts. For example, Santini et al. (2014) found a molecular gas depletion time of 100-300 Myr for a large sample of galaxies using dust continuum measurements from Herschel and found an evolution of the molecular gas depletion time with redshift, by about a factor of 5 from $z \sim 0$ to $z \sim 5$. Scoville et al. (2016) found a characteristic gas depletion time of 200-700 Myr for a sample of galaxies at redshift $z>1$. The entire population of SFGs at $z>1$ has $\sim 2-5$ times shorter gas depletion times than low- $z$ galaxies (Scoville et al. 2016). The gas depletion time of $\sim 107_{-31}^{+44}$ Myr for Galactic massive clumps in our sample seems to be smaller than the characteristic gas depletion time in external galaxies. Interestingly, as discussed in Section 3.4, the 

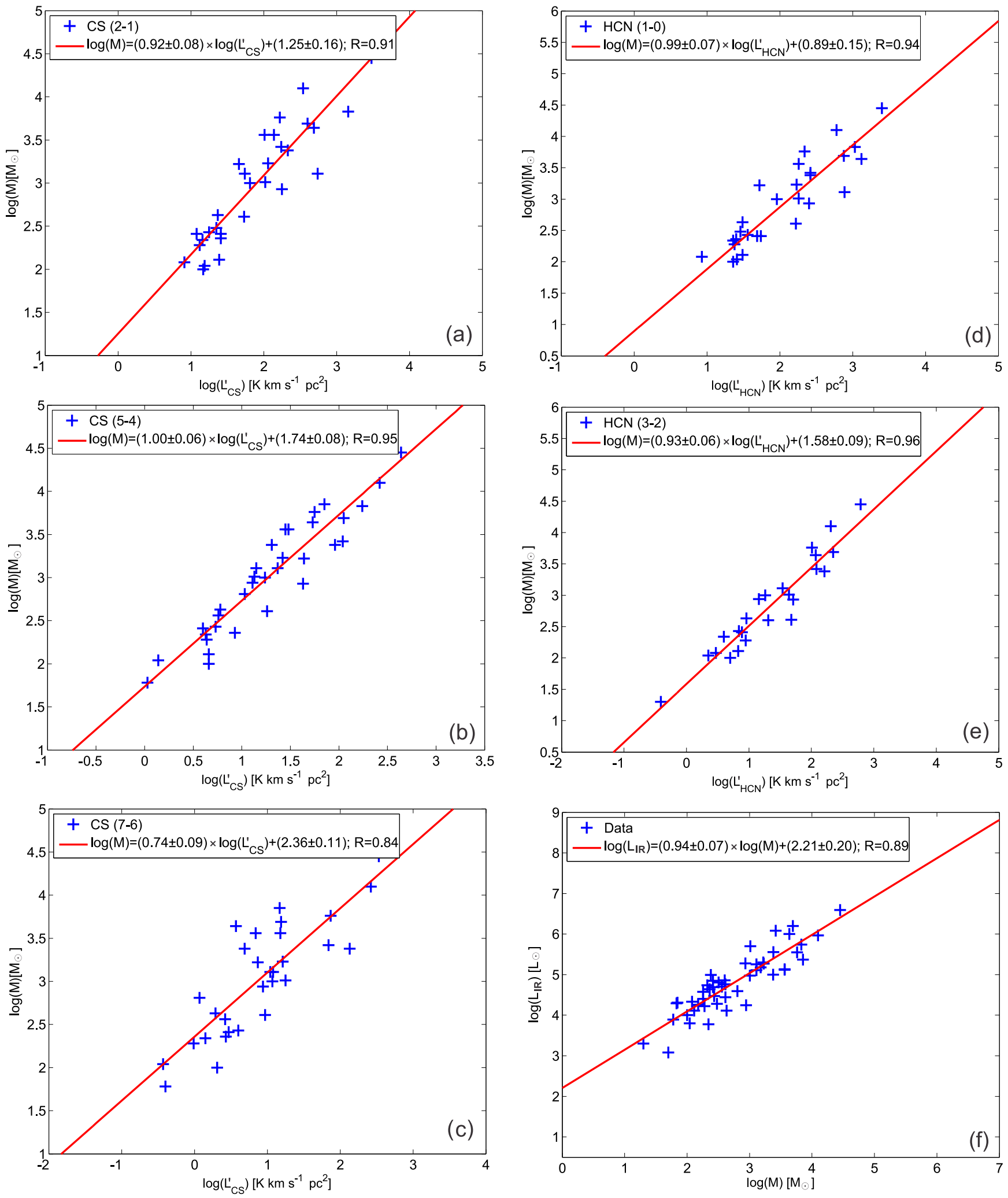

Figure 11. Correlations for the sample of Wu et al. (2010). (a) Clump mass vs. $L^{\prime}{ }_{\mathrm{CS}}$ for CS (2-1) data. (b) Clump mass vs. $L_{\mathrm{CS}}^{\prime}$ for CS (5-4) data. (c) Clump mass vs. $L_{\mathrm{CS}}^{\prime}$ for CS (7-6) data. (d) Clump mass vs. $L_{\mathrm{HCN}}^{\prime}$ for $\mathrm{HCN}$ (1-0) data. (e) Clump mass vs. $L_{\mathrm{HCN}}^{\prime}$ for $\mathrm{HCN}$ (3-2) data. (f) Infrared luminosity vs. clump mass.

integrated Kennicutt-Schmidt laws for Galactic massive clumps can extend to high $z$ SFGs. However dust continuum in extra-galactic studies traces both diffuse dust and dense dust. Therefore the extra-galactic studies can not seperate/resolve any dense gas phase from the total mass. The linear correlation of the integrated Kennicutt-Schemidt laws for both Galactic massive clumps and high $z$ SFGs should indicate that the high $z$ SFGs may have global properties (e.g., density, dense gas fraction, SFEs) at molecular cloud scale similar to Galactic massive clumps at clump scale. In other words, the fraction of 
Table 4

Parameters of Correlations for different Transitions

\begin{tabular}{|c|c|c|c|c|c|c|c|c|c|}
\hline \multirow{2}{*}{ Group } & \multirow{2}{*}{$\begin{array}{c}\mathrm{E}_{u} \\
(\mathrm{~K})\end{array}$} & \multirow{2}{*}{$\begin{array}{c}n_{\text {crit }}{ }^{\mathrm{a}} \\
\left(\mathrm{cm}^{-3}\right)\end{array}$} & \multirow{2}{*}{$\begin{array}{c}n_{\mathrm{eff}}^{\mathrm{b}} \\
\left(\mathrm{cm}^{-3}\right)\end{array}$} & \multicolumn{3}{|c|}{$L_{\mathrm{TIR}}-L_{\mathrm{mol}}^{\prime}{ }^{\mathrm{c}}$} & \multicolumn{3}{|c|}{$M_{\text {clump }}-L_{\text {mol }}^{\prime}$} \\
\hline & & & & $\alpha$ & $\beta$ & $\mathrm{R}$ & $\alpha$ & $\beta$ & $\mathrm{R}$ \\
\hline CS $(2-1)$ & 7.1 & $1.0 \mathrm{E}+5$ & $1.2 \mathrm{E}+4$ & $1.03(0.05)$ & $3.25(0.11)$ & 0.80 & $0.92(0.08)$ & $1.25(0.16)$ & 0.91 \\
\hline CS $(5-4)$ & 35.3 & $1.7 \mathrm{E}+6$ & $2.5 \mathrm{E}+5$ & $1.05(0.05)$ & $3.77(0.08)$ & 0.86 & $1.00(0.06)$ & $1.74(0.08)$ & 0.95 \\
\hline $\mathrm{HCN}(1-0)$ & 4.25 & $3.0 \mathrm{E}+5$ & $4.5 \mathrm{E}+3$ & $1.07(0.06)$ & $2.98(0.14)$ & 0.85 & $0.99(0.07)$ & $0.89(0.15)$ & 0.94 \\
\hline $\mathrm{HCN}(3-2)$ & 25.52 & $1.0 \mathrm{E}+7$ & $7.3 \mathrm{E}+4$ & $0.88(0.06)$ & $3.94(0.11)$ & 0.92 & $0.93(0.06)$ & $1.58(0.09)$ & 0.96 \\
\hline $\mathrm{HCN}(4-3)$ & 42.53 & $2.3 \mathrm{E}+7$ & $3.2 \mathrm{E}+5$ & $0.74(0.06)$ & $4.09(0.09)$ & 0.79 & $0.85(0.04)$ & $2.02(0.05)$ & 0.89 \\
\hline
\end{tabular}

Notes.

${ }^{a}$ Critical density at $20 \mathrm{~K}$, from Shirley \& Yancy (2015)

${ }^{\mathrm{b}}$ Effective excitation density at $20 \mathrm{~K}$, from Shirley \& Yancy (2015).

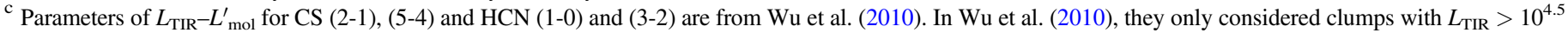
$L_{\odot}$. To compare with Wu et al. (2010), we also only fitted $L_{\mathrm{TIR}}-L_{\mathrm{CS}}^{\prime}$ of $\mathrm{HCN}(4-3)$ and CS (7-6) for clumps with $L_{\mathrm{TIR}}>10^{4.5} L_{\odot}$.
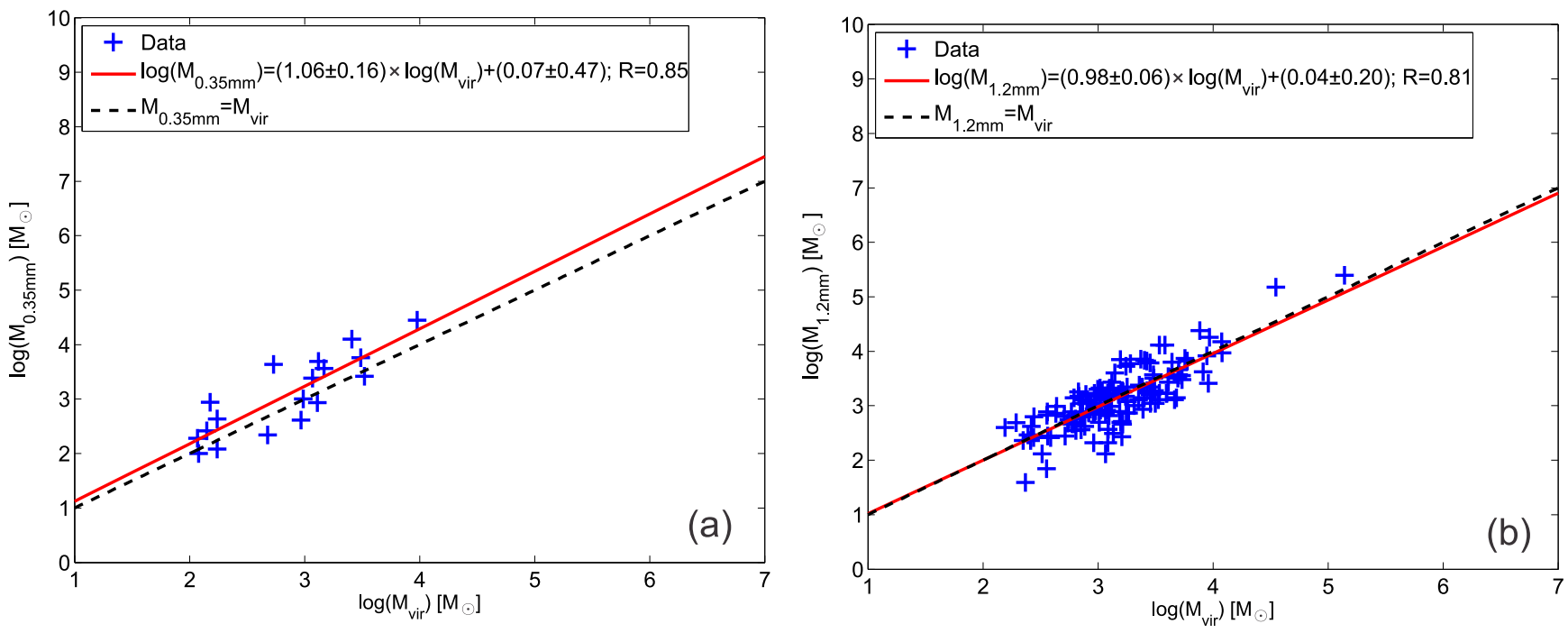

Figure 12. (a) Clump mass from (Mueller et al. 2002) vs. virial mass derived from HCN (3-2) correlations for the sample of Wu et al. (2010). (b) Clump mass derived from $1.1 \mathrm{~mm}$ continuum vs. virial mass derived from CS (7-6) correlations for our sample. The red lines represent the best power law fits. The black dashed lines represent linear correlations.

massive clumps (or dense gas) in molecular clouds of high $z$ SFGs should be much higher than the Milky Way. While the low $z$ galaxies have much lower SFEs than Galactic clumps, suggesting that their molecular clouds contain less massive clumps (or dense gas) than high $z$ SFGs. In high $z$ SFGs, dispersive gas motions (as opposed to ordered rotation) and/or galaxy interactions will lead to compression in the highly dissipative ISM (Scoville et al. 2016), enhancing the density and SFR per unit gas mass in their molecular clouds and creating more massive clumps (or dense gas) than low $z$ galaxies.

\section{SUMMARY}

We carried out HCN (4-3) and CS (7-6) single-point observations, using the ASTE telescope, toward the 146 IRAS sources reported by Faúndez et al. (2004) in order to investigate the K-S law for Galactic clumps. Our main conclusions are:

(1) The SFRs indicated by total infrared luminosities $\left(L_{\mathrm{TIR}}\right)$ are linearly correlated with clump masses for those clumps with $L_{\mathrm{TIR}}>10^{3} L_{\odot}$, leading to a constant gas depletion time of $107_{-31}^{+44}$ Myr. The integrated KennicuttSchmidt laws for Galactic massive clumps can extend to high $z$ star forming galaxies (SFGs). While low $z$ galaxies have much smaller global SFEs (or longer gas depletion time) than Galactic massive clumps and high $z$ SFGs. High $z$ SFGs may have much larger fraction of massive clumps (or dense gas) in molecular clouds than low $z$ galaxies.

(2) The $L_{\mathrm{TIR}}-L_{\text {mol }}^{\prime}$ correlations for $\mathrm{HCN}$ (4-3) and CS (7-6) for clumps are tight and sublinear over about four orders of magnitude in $L_{\mathrm{TIR}}$. We did not notice any breakup of correlations at a luminosity threshold of $10^{4.5} L_{\odot}$ as suggested by $\mathrm{Wu}$ et al. (2010). Instead, the correlations can extend down to clumps with $L_{\mathrm{TIR}}<10^{3} L_{\odot}$. These correlations become linear when taking into account external galaxies.

(3) We find that $L_{\mathrm{TIR}}-L_{\mathrm{CS}}^{\prime}$ and $L_{\mathrm{TIR}}-L_{\mathrm{HCN}}^{\prime}$ correlations for clumps with lower dust temperature, lower luminosity-tomass ratio and higher $\sigma_{\text {line }} / \sigma_{\text {vir }}$ are closer to linear. While the correlations for clumps with higher dust temperature, higher luminosity-to-mass ratio and lower $\sigma_{\text {line }} / \sigma_{\text {vir }}$ tend 
toward sublinear. Such bimodal behavior of $L_{\mathrm{TIR}}-L_{\text {mol }}^{\prime}$ correlations may be due to evolutionary effects. The slopes of $L_{\mathrm{IR}}-L_{\text {mol }}^{\prime}$ correlations (or current star formation law) become more shallow as clumps evolve.

(4) The line luminosities of molecular tracers with upper energies and effective excitation densities lower than the median dust temperature and median volume density of the whole sample are linearly correlated with clump masses. While high effective excitation density tracers cannot linearly trace the total clump masses, leading to a sublinear correlations for both $M_{\text {clump }}-L_{\text {mol }}^{\prime}$ and $L_{\mathrm{TIR}^{-}}$ $L_{\text {mol }}^{\prime}$ relations. Since clump masses traced by dust continuum emission are linearly correlated with infrared luminosities. The (sub)linear $M_{\text {clump }}-L_{\text {mol }}^{\prime}$ correlations can naturally explain the (sub)linear $L_{\mathrm{TIR}}-L_{\text {mol }}^{\prime}$ correlations (i.e., the $\mathrm{K}-\mathrm{S}$ law) for different molecular line tracers.

We are grateful to the ASTE staff. The ASTE telescope is operated by National Astronomical Observatory of Japan (NAOJ). Tie Liu is supported by KASI fellowship. Y. Wu is partly supported by the China Ministry of Science and Technology under State Key Development Program for Basic Research (No.2012CB821800), the grants of NSFC No.11373009 and No.11433008. Ke Wang acknowledges the support from ESO fellowship and DFG Priority Program 1573 ("Physics of the Interstellar Medium") grant WA3628-1/1. This work was carried out in part at the Jet Propulsion Laboratory, operated for NASA by the California Institute of Technology. S. L. Qin is supported by NSFC under grant No. 11373026, and Top Talents Program of Yunnan Province (2015HA030). MJ acknowledges the support of the Academy of Finland Grant No. 1285769. LB acknowledges support from CONICYT grant PFB06. JEL was supported by the Basic Science Research Program through the National Research Foundation of Korea (NRF) (grant No. NRF-2015R1A2A2A01004769) and the Korea Astronomy and Space Science Institute under the R\&D program (Project No. 20151-32018) supervised by the Ministry of Science, ICT, and Future Planning. LVT acknowledges the support by the OTKA grants K101393 and NN-111016. The anonymous referee provided very insightful comments.

\section{REFERENCES}

Clements, D. L., Dunne, L., \& Eales, S. 2010, MNRAS, 403, 274

Clemens, M. S., Negrello, M., De Zotti, G., et al. 2013, MNRAS, 433, 695

Csengeri, T., Weiss, A., Wyrowski, F., et al. 2016, A\&A, 585, 104

da Cunha, E., Eminian, C., Charlot, S., et al. 2010, MNRAS, 403, 1894

Daddi, E., Elbaz, D., Walter, F., et al. 2010, ApJL, 714, L118

Dunne, L., Eales, S., Edmunds, Mi., et al. 2000, MNRAS, 315, 115

Faúndez, S., Bronfman, L., Garay, G., et al. 2004, A\&A, 426, 97

Gao, Y., \& Solomon, P. M. 2004, ApJ, 606, 271

Genzel, R., Tacconi, L. J., Gracia-Carpio, J., et al. 2010, MNRAS, 407, 2091

Graciá-Carpio, J., García-Burillo, S., Planesas, P., et al. 2008, A\&A, 479, 703

Greve, T. R., Leonidaki, I., Xilouris, E. M., et al. 2014, ApJ, 794, 142

Guilloteau, S., \& Lucas, R. 2000, in ASP Conf. Ser. 217, Imaging at Radio through Submillimeter Wavelengths, ed. J. G. Mangum \& S. J. E. Radford, (San Francisco, CA: ASP), 299

Hjorth, J., Gall, C., Michałowski, \& MichałJ, J. 2014, ApJ, 782L, 23

Juneau, S., Narayanan, D. T., Moustakas, J., et al. 2009, ApJ, 707, 1217

Kelly, B. C. 2007, ApJ, 665, 1489

Kennicutt, R. C., Jr. 1998, ApJ, 498, 541

Kennicutt, R. C., \& Evans, N. J. 2012, ARA\&A, 50, 531

Lada, C. J., Forbrich, J., Lombardi, M., et al. 2012, ApJ, 745, 190

Lada, C. J., Lombardi, M., \& Alves, J. F. 2010, ApJ, 724, 687

Lada, C. J., Lombardi, M., Roman-Zuniga, C., et al. 2013, ApJ, 778, 133

Liu, D., Gao, Y., Isaak, K., et al. 2015, ApJ, 810L, 14L

Liu, T., Wu, Y., \& Zhang, H. 2013, ApJL, 775, L2

Ma, B., Tan, J. C., \& Barnes, P. J. 2013, ApJ, 779, 79

Magdis, G. E., Rigopoulou, D., Helou, G., et al. 2013, A\&A, 558, 136

Molinari, S., Merello, M., Elia, D., et al. 2016, ApJL, 826L, 8

Mueller, K. E., Shirley, Y. L., Evans, N. J., II, et al. 2002, ApJS, 143, 469

Narayanan, D., Cox, T. J., Shirley, Y., et al. 2008, ApJ, 684, 996

Ossenkopf, V., Rl'lig, M., Simon, R., et al. 2010, A\&A, 518, L79

Reiter, M., Shirley, Y. L., Wu, J., et al. 2011, ApJS, 195, 1

Rowlands, K., Dunne, L., Maddox, S., et al. 2012, MNRAS, 419, 2545

Santini, P., Maiolino, R., Magnelli, B., et al. 2014, A\&A, 562, A30

Schmidt, M. 1959, ApJ, 129, 243

Scoville, N., Sheth, K., Aussel, H., et al. 2016, ApJ, 820, 83

Shirley, \& Yancy, L. 2015, PASP, 127, 299

Stephens, I. W., Jackson, J. M., \& Whitaker, J. S. 2016, ApJ, 824, 29

van Kempen, T. A., van Dishoeck, E. F., Güsten, R., et al. 2009, A\&A, 501,633

Vutisalchavakul, N., \& Evans, N. J., II 2013, ApJ, 765, 129

Wienen, M., Wyrowski, F., Menten, K. M., et al. 2015, A\&A, 579, 91

Williams, J. P., de Geus, E. J., \& Blitz, L. 1994, ApJ, 428, 693

Wu, J., Evans, N. J., II, Gao, Y., et al. 2005, ApJL, 635, L173

Wu, J., Evans, N. J., II, Shirley, Y. L., et al. 2010, ApJS, 188, 313

Zhang, Z.-Y., Gao, Y., Henkel, C., et al. 2014, ApJL, 784, L31

Zhou, S., Evans, N. J., II, Koempe, C., \& Walmsley, C. M. 1993, ApJ, 404,232 